\title{
Weak-line T Tauri stars: circumstellar disks and companions ${ }^{\star}$
}

\section{Spectral energy distributions and infrared excesses}

\author{
À. Gras-Velázquez and T. P. Ray
}

Dublin Institute for Advanced Studies, 5 Merrion Square, Dublin 2, Ireland

e-mail: agv@cp.dias.ie

Received 19 November 2004 / Accepted 11 August 2005

\section{ABSTRACT}

We have analysed new infrared photometric data taken by the Infrared Space Observatory, in combination with 2MASS data where available, of 12 weak-line T Tauri stars (WTTS), belonging to different molecular clouds. A control study of 6 classical T Tauri stars (CTTS) for comparison with IRAS data has also been made. WTTS traditionally are not expected to have any circumstellar material, but we have found that 11 of the WTTS studied, have a spectral energy distribution that suggests the presence of reprocessing circumstellar disks, albeit half of them with apparent inner "dust holes". The spectral energy distribution curves of all the stars in our sample show infrared excess in all cases except one. We have also searched for companions to these stars and studied their possible contributions to the infrared emission. We find that 10 of the WTTS with excess infrared emission have companions that may contribute measurably and, in some cases significantly, at infrared wavelengths. In the case of the binary systems, the amount of excess due to the companion is still unclear but it is unlikely they are the only source of all the observed excess. We give estimations of the excesses and masses of the stars and also find their ages and radii to be in agreement with those expected for T Tauri stars. Our results suggest there is a continuum in infrared excess properties from CTTS to WTTS rather than a clear differentiation based on a somewhat arbitrary $E W(\mathrm{H} \alpha)$ criterion.

Key words. stars: formation - infrared: stars - stars: pre-main sequence - circumstellar matter - techniques: photometric

\section{Introduction}

Circumstellar disks are common around low-mass $\left(M \sim 1 M_{\odot}\right)$ pre-main sequence stars, e.g. Meyer, et al. (1997), and references therein; Kenyon \& Hartmann (1990). These disks emit most of their energy in the infrared. The infrared emission is the result of the disk's reprocessing of the radiation emitted by the stellar photosphere, see for example, Wood et al. (2002) or Chandler (1998) and in some cases, viscous accretion that heats the disk (Kenyon \& Hartmann 1987). As a result, the presence of disks around pre-main sequence stars can be detected by an excess in the infrared wavelengths over the spectral energy distribution (SED) corresponding to the star's photosphere (Kenyon \& Hartmann 1987).

$\mathrm{T}$ Tauri stars are pre-main sequence (no older than $\left.10^{5}-10^{7} \mathrm{yr}\right)$, low-mass $\left(0.2 \leq M_{*} / M_{\odot} \leq 2\right)$ stars generally divided in two subgroups: classical T Tauri Stars (CTTS) and weak-line T Tauri Stars (WTTS), e.g. Martín (1998). As strong $\mathrm{H} \alpha$ line emission is believed to be an indicator of the presence of an accretion disk (see Kenyon \& Hartmann 1990; and references therein), authors have traditionally stated that CTTS are stars with $\mathrm{H} \alpha$ equivalent widths, $E W(\mathrm{H} \alpha)$, greater than $10 \AA$ and large infrared excesses (indicative of circumstellar

\footnotetext{
^ Based on observations taken by ISO.
}

disks), while WTTS have $E W(\mathrm{H} \alpha) \leq 10 \AA$ and almost no excess (Alcalá et al. 1993). This fact has led to the suggestion that WTTS are more evolved CTTS, e.g. Duvert et al. (2000). More recently, categorization by fixed $E W(\mathrm{H} \alpha)$ has been questioned and a weak dependence of the $E W(\mathrm{H} \alpha)$ borderline value on spectral type has been invoked (Martín 1998).

Thanks to the improved sensitivity and angular resolution of the Infrared Space Observatory (ISO) over the Infrared Astronomical Satellite (IRAS), ISO could detect much fainter infrared emission from Young Stellar Objects (YSOs). In particular we were able to obtain infrared photometry of a number of $\mathrm{T}$ Tauri stars at wavelengths for which they have not been previously observed.

Another characteristic of the formation of low-mass stars seems to be a high probability of them being born in multiple systems (Geoffray \& Monin 2001; Günther \& Kley 2002). The aperture of ISO's photometer, ISOPHOT, ranged from 10" to $120^{\prime \prime}$ for the observations referred to here. Therefore, any infrared excess could potentially derive from an embedded companion (Chelli et al. 1988) and we investigate that possibility here.

Using UV, optical and near-infrared data from the literature and the 2MASS and DENIS surveys, along with our new ISOPHOT photometry, we have constructed spectral energy 
Table 1. Pre-main sequence stars observed.

\begin{tabular}{|c|c|c|c|c|c|c|c|c|}
\hline Star & Location & Ref. & $\operatorname{TTS}^{a}$ & TDTnum $^{b}$ & RA (2000) & $\operatorname{Dec}(2000)$ & $\mathrm{HBC}$ & IRAS \\
\hline T Cha & Dcld 300.2-16.9 & 1 & $\mathrm{w}$ & 11700504 & 115713.53 & -792131.5 & 591 & IRAS 11547-7904 \\
\hline Sz 68 & Lup 1 & 2 & $\mathrm{w}$ & 11801413 & 154512.87 & -341730.6 & 248 & IRAS $15420-3408$ \\
\hline RU Lup & Lup 2 & 1 & $\mathrm{c}$ & 11801914 & 155642.31 & -374915.5 & 251 & IRAS $15534-3740$ \\
\hline EX Lup & Lup 3 & 3 & $\mathrm{c}$ & 11801715 & 160305.48 & -401825.9 & 253 & IRAS $15597-4010$ \\
\hline $\mathrm{Sz} 124$ & Lup 3 & 4 & $\mathrm{w}$ & 11801816 & 161153.35 & -390215.7 & 631 & - \\
\hline ROX 2 & $\rho$ Oph / B42 & $4 / 5$ & $\mathrm{w}$ & 12101218 & 162525.00 & -235530.0 & 635 & - \\
\hline DoAr 24 & $\rho$ Oph / B42 & $4 / 5$ & $\mathrm{c}$ & 12101320 & 162617.20 & -242023.0 & 638 & - \\
\hline DoAr 24E & $\rho$ Oph / B42 & 5 & $\mathrm{w}$ & 12001722 & 162623.40 & -242102.0 & 639 & - \\
\hline SR 12 & $\rho$ Oph / B42 & 5 & $\mathrm{w}$ & 12101023 & 162719.50 & -244140.1 & 263 & - \\
\hline ROX 31 & $\rho$ Oph / B42 & 5 & $\mathrm{w}$ & 12002025 & 162752.00 & -244048.0 & 642 & - \\
\hline Wa Oph/4 & $\mathrm{L} 162=\mathrm{LDN} 162$ & 6 & $\mathrm{w}$ & 11400526 & 164818.00 & -141115.0 & 652 & - \\
\hline Wa Oph/6 & L162 / Oph & 6 & $\mathrm{c}$ & 11400627 & 164845.69 & -141634.8 & 653 & IRAS 16459-1411 \\
\hline IX Oph & B59 & 1 & $\mathrm{w}$ & 12101729 & 170948.16 & -271659.3 & 272 & - \\
\hline V1725 Oph & B62 & 4 & $\mathrm{w}$ & 11801330 & 171613.85 & -205745.7 & 661 & - \\
\hline CoKu Ser G1 & L572 / Ser MC $L_{\text {exc. }}$ & $4 / 7$ & $\mathrm{w}$ & 11401333 & 182815.19 & -000241.6 & 667 & - \\
\hline CoKu Ser G7 & L572 & 4 & $\mathrm{w}$ & 11401535 & 182956.56 & +011449.1 & 672 & IRAS $18274+0112$ \\
\hline V536 Aql & - & - & $\mathrm{c}$ & 13000939 & 193857.00 & +103018.0 & 294 & IRAS $19365+1023$ \\
\hline MacC H3 & Cep OB4 & 4 & $\mathrm{c}$ & 13100944 & 235426.80 & +665421.0 & 319 & - \\
\hline
\end{tabular}

Notes to table: ${ }^{a}$ See Table 3 for corresponding $E W(\mathrm{H} \alpha)$ and references. Classification w and c refer to WTTS and CTTS respectively. ${ }^{b}$ TDTnum is the assigned ISO observation number.

References: (1) Bertout et al. (1999), (2) Geoffray \& Monin (2001), (3) Appenzeller et al. (1983), (4) Herbig-Bell Catalogue (HBC), (5) Bouvier \& Appenzeller (1992), (6) Walter (1986), (7) Jensen et al. (1996).

distributions for 12 WTTS and 6 CTTS to search for infrared excesses, and where detected, to examine if this emission could be attributed to a disk around the primary target. With this aim in mind, we have also checked for companions and tried to assess their likely contrbution to the excess. The CTTS were included as a control sample for comparison with IRAS data. The ISO data also helps to improve their SEDs for modelling purposes.

Finally, using a H-R diagram with evolutionary tracks and isochrones from Palla \& Stahler (1999), we have obtained the masses and ages for all the stars of our sample.

In this paper we present: in Sect. 2, the justification for our selected sample and technique used to reduce the data; Sect. 3, the procedure to obtain the SED and IR excesses and the complete list of stars with notes discussing particular problems encountered; Sect. 4, the discussion of the IR excesses, masses and ages calculated; and in Sect. 5, a summary of the results obtained. In the Appendix one can find the reduced ISO data as well as optical photometry obtained in La Palma for this paper. A further paper (Gras-Velázquez et al. 2005) will be devoted to detailed modelling of the SEDs in terms of flared disks and the different stars in each system.

\section{Observations}

The WTTS and the CTTS were largely chosen from the Lupus, $\rho$ Ophiuchi and Serpens star forming regions. These stars were selected from the Herbig-Bell Catalog (HBC) according to their $\mathrm{H} \alpha$ emission. A list of the stars, with their coordinates can be found in Table 1. The corresponding IRAS point sources are also included, where known.
The infrared photometry was carried out using the multi-band, multi-aperture photometer PHT-P of ISOPHOT (PHT03), the imaging photo-polarimeter on ISO. Observations for each star were made with 9 filters, with central wavelengths ( $F W H M$ width) of 4.8 (1.55), 7.7 (0.84), 10 (1.86), 12 (6.51), 12.8 (2.33), 15 (2.86), 20 (9.43), 60 (25.48) and $100(40.15) \mu \mathrm{m}$. For the first five wavelengths the aperture used was $10^{\prime \prime}$, while for the 15 and $20 \mu \mathrm{m}$ filters it was $23^{\prime \prime}$ and for the 60 and $100 \mu \mathrm{m}$ filters $120^{\prime \prime}$.

The data was reduced using PIA (ISOPHOT's Interactive Analysis package) V10.0 following the Ramps Pattern Mode for Chopped Measurements and applying the standard procedures given in the manual (Gabriel et al. 1996). The reduced ISO photometry can be found in Appendix A.

The positive flux offset seen in some SEDs at $12.8 \mu \mathrm{m}$ and a negative offset at $20 \mu \mathrm{m}$, are not special features, but systematic flux offsets reported in the ISO handbook for these filters which we have left unaltered here.

In the SEDs of the stars T Cha, Sz 68 and V536 Aql, the flux obtained at $4.8 \mu \mathrm{m}$ appears somewhat less than that from $M$-band ground-based photometry. For T Cha, we have compared this flux at $4.8 \mu \mathrm{m}$ with those from ground-based photometry and ISO's multi-aperture photometric observations (PHT04), and believe the differences are due to temporal variability rather than an instrumental effect. This variability in the near-infrared was already pointed out by Alcalá et al. (1993). The flux in the M band for Sz 68 and V536 Aql, was taken from Geoffray \& Monin (2001). We believe the discrepancy at $5 \mu \mathrm{m}$ for these two stars, is due also to variability, like in the case of $\mathrm{T}$ Cha. Furthermore, for T Cha, we also have MSX photometry 
Table 2. References for the uncorrected photometry used in Figs. 1 to 6 .

\begin{tabular}{lccc}
\hline \hline Star & $U B V$ & $(R I)_{\mathrm{c}}$ & $J H K L$ \\
\hline T Cha & 1 & 1 & $1 / 2$ \\
Sz 68 & 3 & 4 & $2 / 5$ \\
RU Lup & 6 & 6 & $2 / 6$ \\
EX Lup & $7 / 8$ & $8 / 9$ & $2 / 8$ \\
Sz 124 & 10 & 8 & $2 / 8$ \\
ROX 2 & 11 & 11 & 2 \\
DoAr 24 & 11 & 11 & $2 / 12$ \\
DoAr 24E & 11 & $13^{k c}$ & $2 / 12$ \\
SR 12 & 12 & $13^{k c}$ & $2 / 12$ \\
ROX 31 & 11 & 11 & $2 / 14$ \\
Wa Oph/4 & 15 & $16 / 17$ & 2 \\
Wa Oph/6 & 15 & - & 2 \\
IX Oph & 4 & 4 & $2 / 18$ \\
V1725 Oph & 19 & - & 2 \\
CoKu Ser G1 & 20 & 20 & 2 \\
CoKu Ser G7 & $20 / 21$ & 20 & 2 \\
V536 Aq1 & 20 & 20 & $2 / 5$ \\
MacC H3 & 3 & $16 / 20$ & 2 \\
\hline
\end{tabular}

Notes to Table: $\mathrm{kc}=$ photometry in the Cousins system, after being transformed from the Kunkel and Rydgren system using the relationships in Bessell (1979).

References: (1) Alcalá et al. (1993), (2) 2MASS, (3) Herbig-Bell Catalogue (HBC), (4) Covino et al. (1992), (5) Geoffray \& Monin (2001), (6) Gahm et al. (1993), (7) Mundt \& Bastian (1980), (8) Hughes et al. (1994), (9) Herbig et al. (1992), (10) Schwartz \& Noah (1978), (11) Bouvier \& Appenzeller (1992), (12) Rydgren et al. (1976), (13) Rydgren (1980), (14) Ageorges et al. (1997), (15) Walter (1986), (16) USNO-A2.0, (17) DENIS (18) Glass \& Penston (1974), (19) Reipurth \& Gee (1986), (20) Observations for this paper taken using the Isaac Newton Telescope on La Palma, (21) Cohen \& Kuhi (1979).

at $8.3 \mu \mathrm{m}$ (Kraemer et al. 2003) which confirms the ISO results at $7.7 \mu \mathrm{m}$ (see Fig. 1, cross at $8.3 \mu \mathrm{m}$ ).

The rest of the photometry included in the SED curves, was taken from different authors (see the complete list of references in Table 2) as well as the 2MASS and DENIS surveys (the full compilation of the photometry used for the SEDs can be found in Appendix B). For the stars CoKu Ser G1, CoKu Ser G7, V536 Aql and MacC H3, we obtained some photometric data from observations taken in La Palma in November 2003, with the Wide Field Camera on the Isaac Newton Telescope. This was reduced using the astronomical source extractor (SExtractor) from Bertin \& Arnouts (1996) and corrected for airmass and exposure time. This latter photometry can also be found in Appendix A.

\section{Results}

\subsection{Spectral energy distributions}

We present SED graphs (Figs. 1 to 6) for our sample in which we have combined: (squares) optical (UBVRI) and nearinfrared $(J H K L)$ photometry from previous papers and the 2MASS and DENIS surveys, corrected for extinction using the
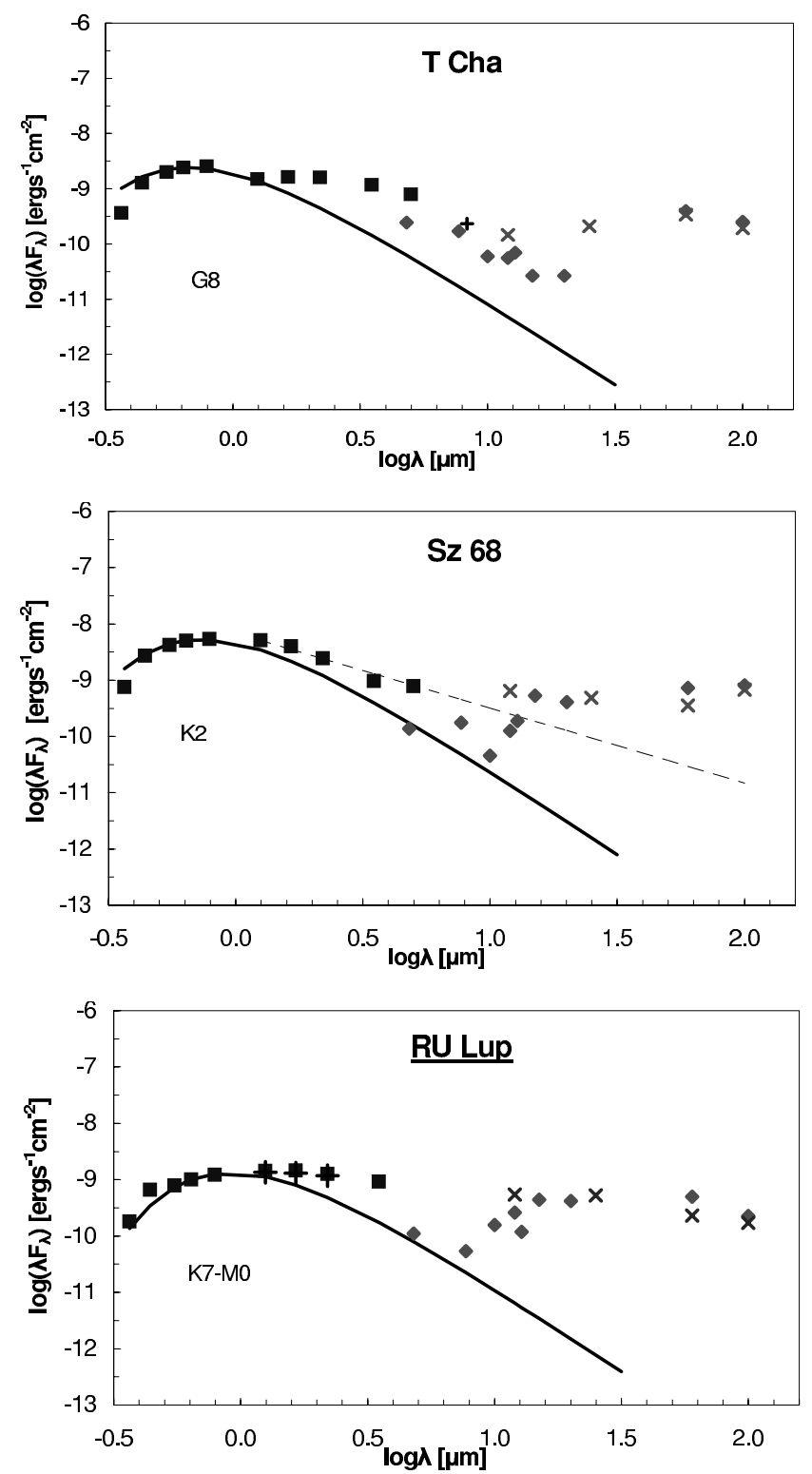

Fig. 1. SEDs of T Cha, Sz 68 and RU Lup. The photometry has been corrected for extinction using the corresponding optical extinction $A_{\mathrm{V}}$ given in Table 3. In this figure, photometric points from the literature and the 2MASS survey are given as filled squares, ISO points as filled diamonds and IRAS points as black x's. A typical star plus flat reprocessing disk fit to the spectra is shown in the case of Sz 68 as a dashed line. The presumed spectral type is also listed. Stars with their name underlined are known CTTS. Other symbols used are explained in the notes for the individual objects.

most reliable value for $A_{\mathrm{V}}$ (when consistency between 2MASS and the $J H K$ photometry presented in the literature was found, $L$ and $M$-band photometry were also taken from the same paper, if available); (diamonds) new ISO photometry reduced by us for this paper; (x's) IRAS values when available obtained from SIMBAD and Weaver \& Jones (1992); and (continuous curve) the black body distribution representing the stellar photosphere, dependent on the spectral type. The crosses and discontinuous curves are the corresponding photometry and black body when a different temperature or an alternative extinction, 
À. Gras-Velázquez and T. P. Ray: Weak-line T Tauri stars: circumstellar disks and companions. I.
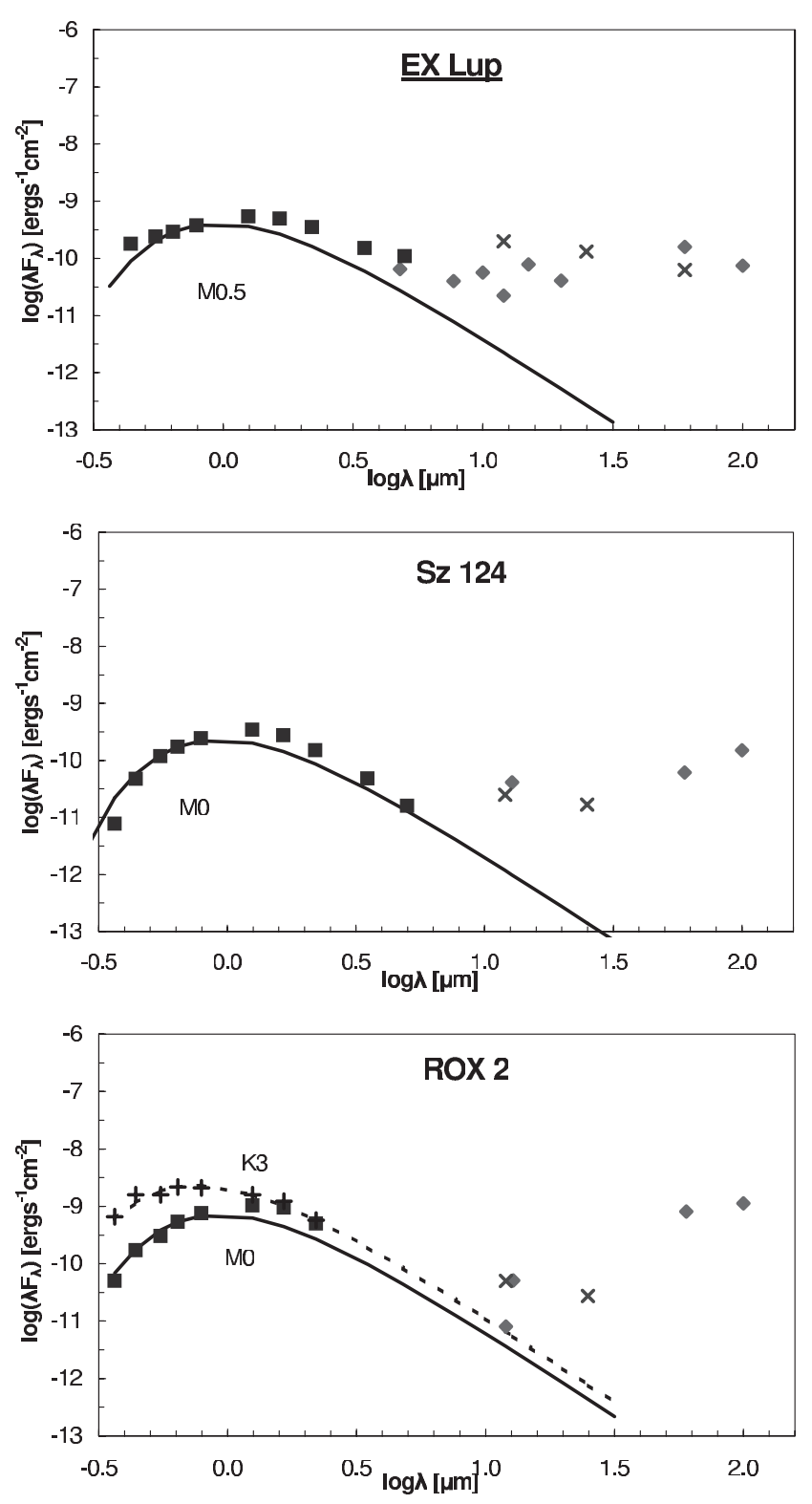

Fig. 2. SEDs (cont.) of EX Lup, Sz 124 and ROX 2, as in Fig. 1.

was suggested in the literature (see details for each case in the corresponding notes on individual objects). The discontinuous straight line, present in $\mathrm{Sz} 68$, as an example, represents the expected SED from a geometrically-thin, optically thick reprocessing disk viewed face-on $\left(\lambda F_{\lambda} \sim \lambda^{-4 / 3}\right.$ at $\lambda \geq 2.2 \mu \mathrm{m}$, Hillenbrand et al. 1992). This line has been normalized to the flux at the $J$-band, as emission at this wavelength is considered to come only from the star, for flat disks without dust holes. The flatter slope we typically find in the infrared energy distribution is more in agreement with flared reprocessing disk models as suggested by Kenyon \& Hartmann (1987), although a more exhaustive comparison remains to be done (Gras-Velázquez et al. 2005).

The combined SEDs of the binary systems for which we have NIR photometry for the companion can be found in Figs. 7 to 14 .
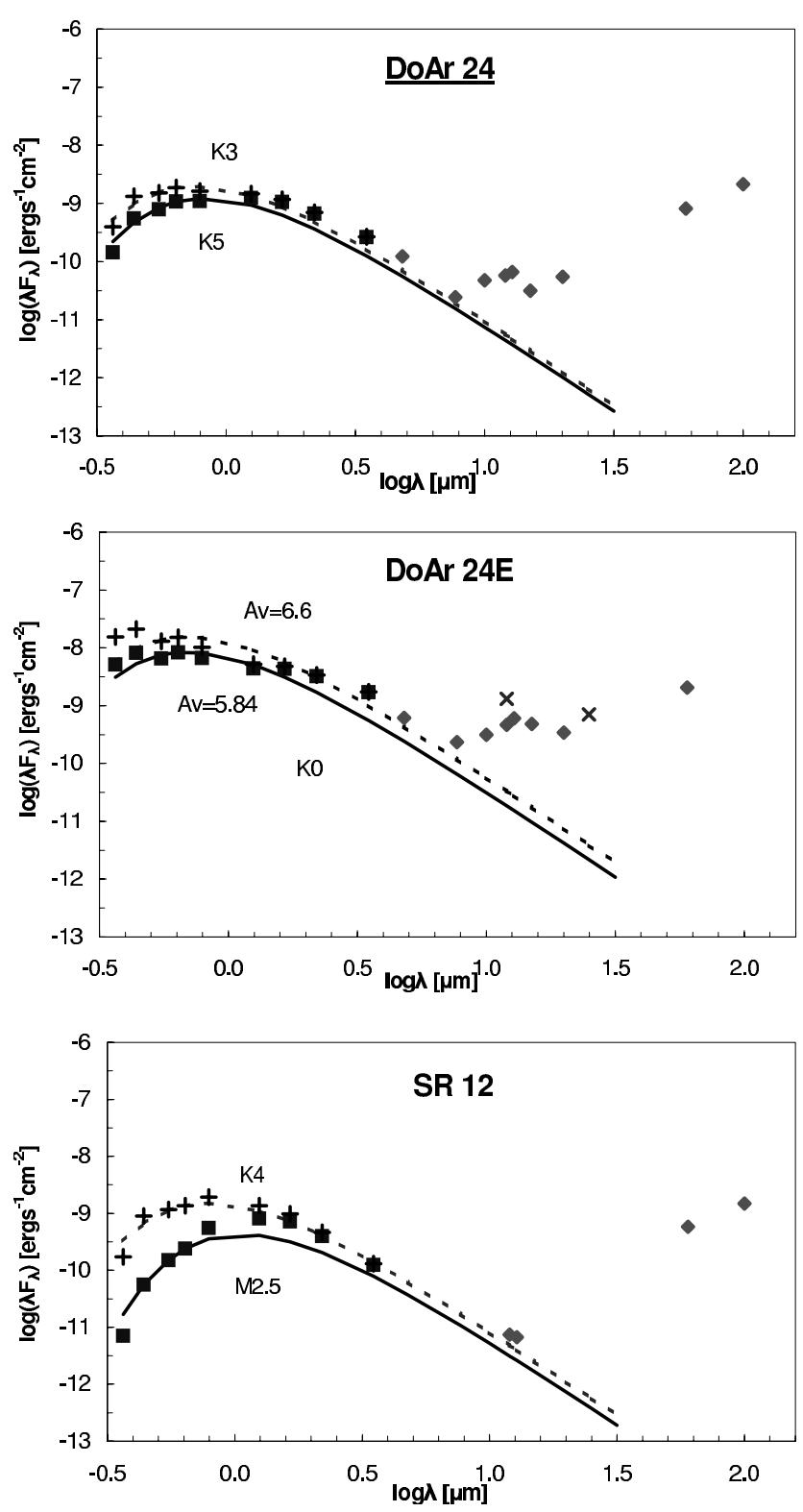

Fig. 3. SEDs (cont.) of DoAr 24, DoAr 24E and SR 12, as in Fig. 1.

We have assigned w (WTTS) or c (CTTS) to the stars in Table 3 adopting the classification scheme suggested by Martín (1998), with the $E W(\mathrm{H} \alpha)$ threshold depending on the spectral type. Stars with the following $E W(\mathrm{H} \alpha)$ are considered WTTS:

$E W(\mathrm{H} \alpha) \leq 5 \AA$ (for K-type stars)

$E W(\mathrm{H} \alpha) \leq 10 \AA$ (for M0-M2 stars)

$E W(\mathrm{H} \alpha) \leq 20 \AA$ (for later M stars)

Note that stars with their names underlined in the SED graphs are CTTS.

\subsection{Approach to the data analysis}

The visual extinction $\left(A_{\mathrm{V}}\right)$ was obtained assuming $A_{\mathrm{V}}=R_{V} \cdot E_{B-V} \quad$ (Chini \& Wargau 1998), where $E_{B-V}$ is the $B-V$ colour excess. The adopted colours and bolometric corrections are from Reid (2002) and Lang (1992), respectively. $R_{V}$ for members of the $\rho$ Ophiuchi Dark Cloud was 

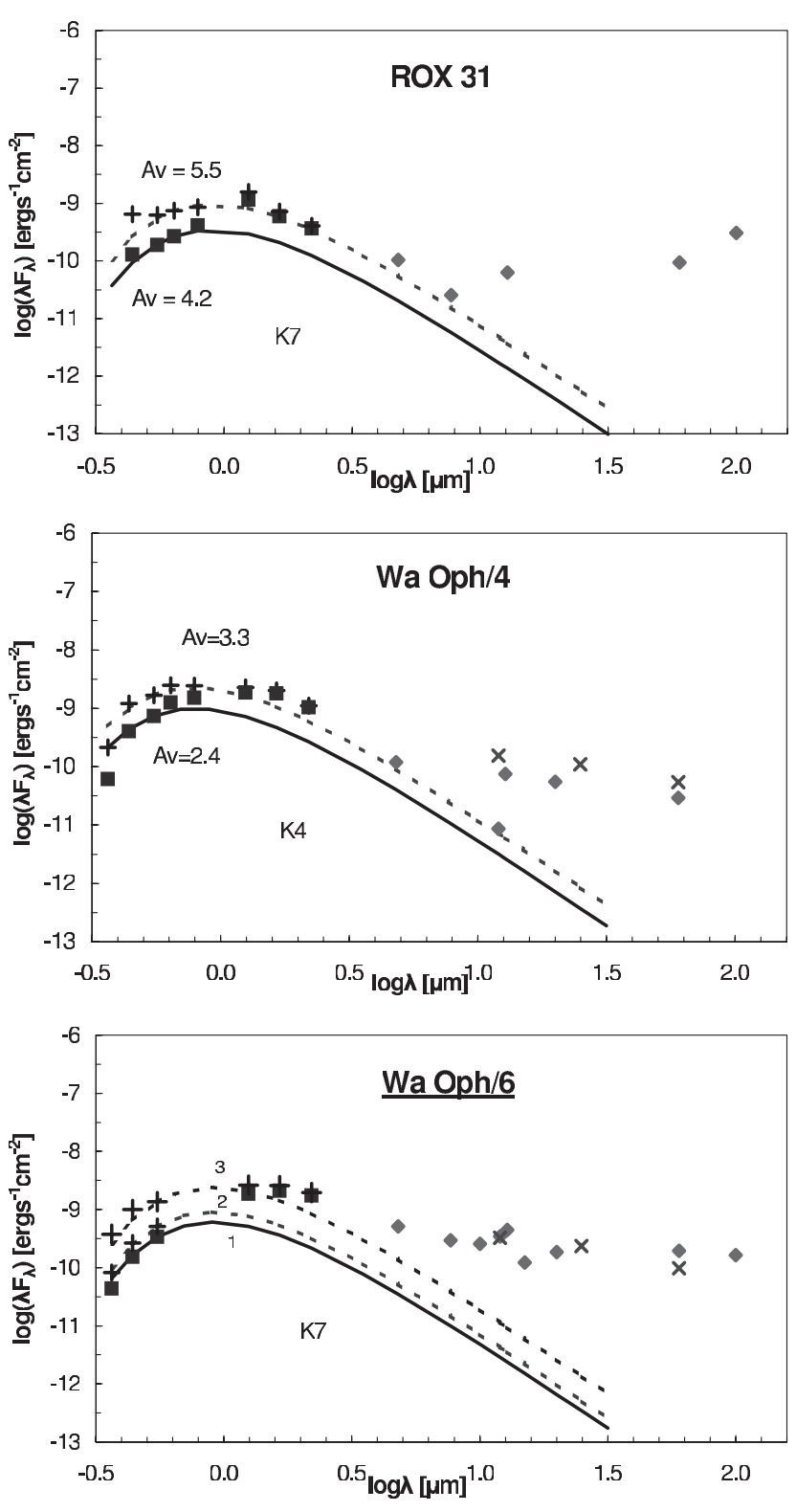

Fig. 4. SEDs (cont.) of ROX 31, Wa Oph/4 and Wa Oph/6, as in Fig. 1.

taken to be 4.2 if $E_{V-K} \geq 1.0$ (Rydgren 1980; Teixeira \& Emerson 1999). Otherwise the standard ISM value for $R_{V} \simeq$ 3.1 , was assumed. As it is difficult to know the exact value of the extinction in a number of cases we have taken the approach of assuming the highest possible value of $A_{\mathrm{V}}$ for each star. This procedure may lead to an overestimate of the photospheric luminosity but the infrared emission will remain largely unaltered. Having higher stellar luminosity and the same infrared emission, decreases the ratio between them. Hence, the calculated infrared excesses, expressed in terms of the stellar luminosity, will be lower limits (Finkenzeller \& Basri 1987).

The excesses in the infrared emission have been calculated following the method already used by Cabrit et al. (1990), where the infrared excess $I_{\text {exc. }}$ is the ratio of $\left(L_{\mathrm{IR}}-L_{\mathrm{bb}}\right)$ and the luminosity of the star, $L_{*}$. $L_{\mathrm{IR}}$ is the infrared luminosity obtained by adding: 1 . the flux calculated, from the wavelength
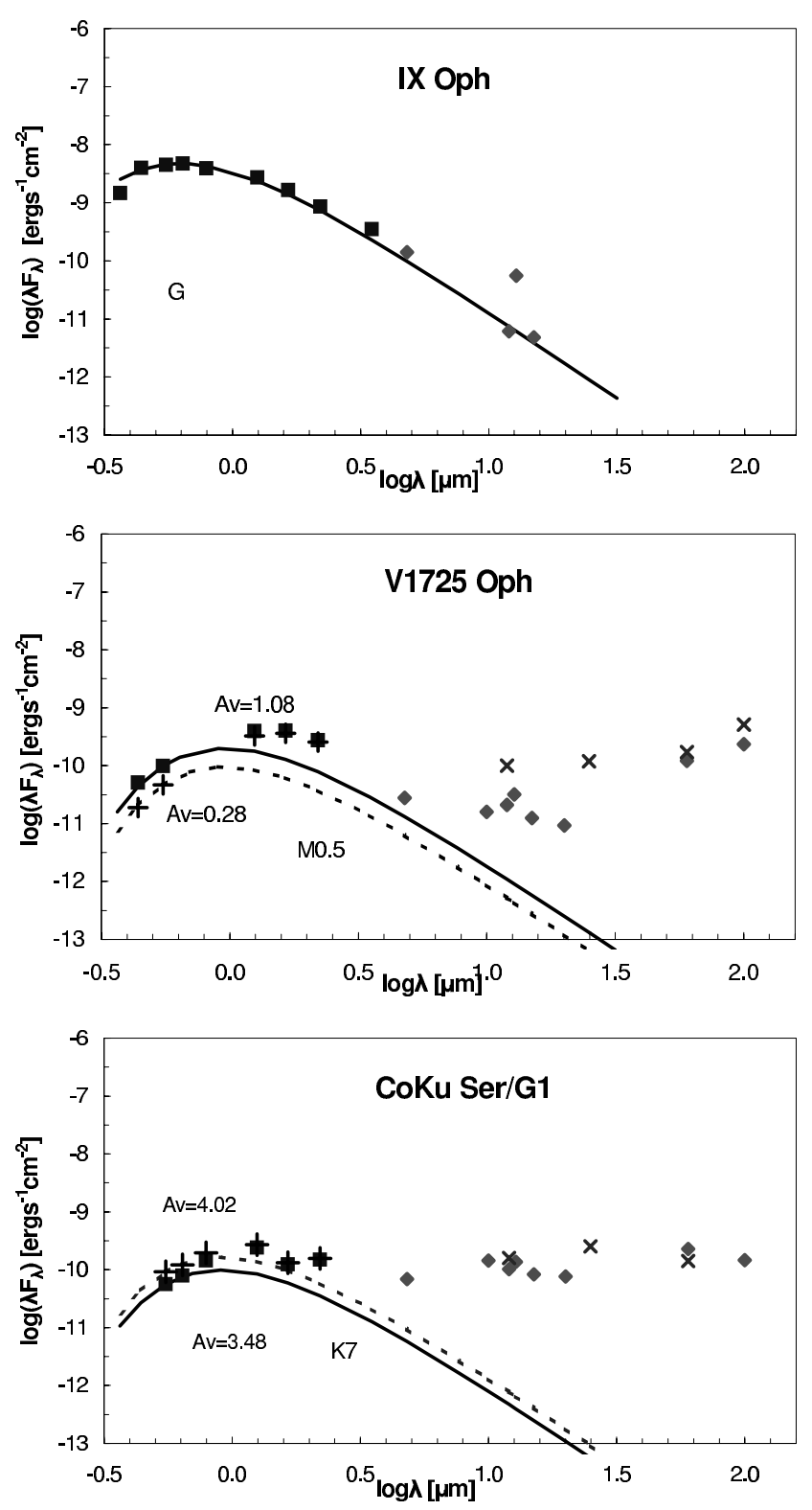

Fig. 5. SEDs (cont.) of IX Oph, V1725 Oph and CoKu Ser G1, as in Fig. 1.

stated for each star in Table 3 to the longest wavelength available, by trapezoidal integration, and 2. the flux from the longest wavelength obtained by ISO for each star to infinite wavelengths using the expression from Chavarría (1981), which supposes the emission at this wavelength to be the peak of a black body curve. $L_{\mathrm{bb}}$ is the luminosity of the black body representing the stellar photosphere, calculated over the same interval as $\mathrm{L}_{I R}$, from integration of the Rayleigh-Jeans part of the spectrum. The bolometric luminosity of the star was obtained using $L_{*}=3.02 \times 10^{35} \times 10^{-0.4\left(B C+M_{\mathrm{v}}\right)}\left(\mathrm{erg} \mathrm{s}^{-1}\right)$, where $\mathrm{BC}$ is the bolometric correction and $M_{\mathrm{v}}$ is the absolute visual magnitude. It is important to note that the flat reprocessing geometrically thin disk model permits an infrared excess no larger than $0.25 L_{*}$ (Kenyon \& Hartmann 1987; Hillenbrand et al. 1992).

In all cases, we also state the luminosity of the infrared excess, $L_{\text {exc. }}$, i.e. $L_{\mathrm{IR}}-L_{\mathrm{bb}}$, in $L_{\odot}$. 

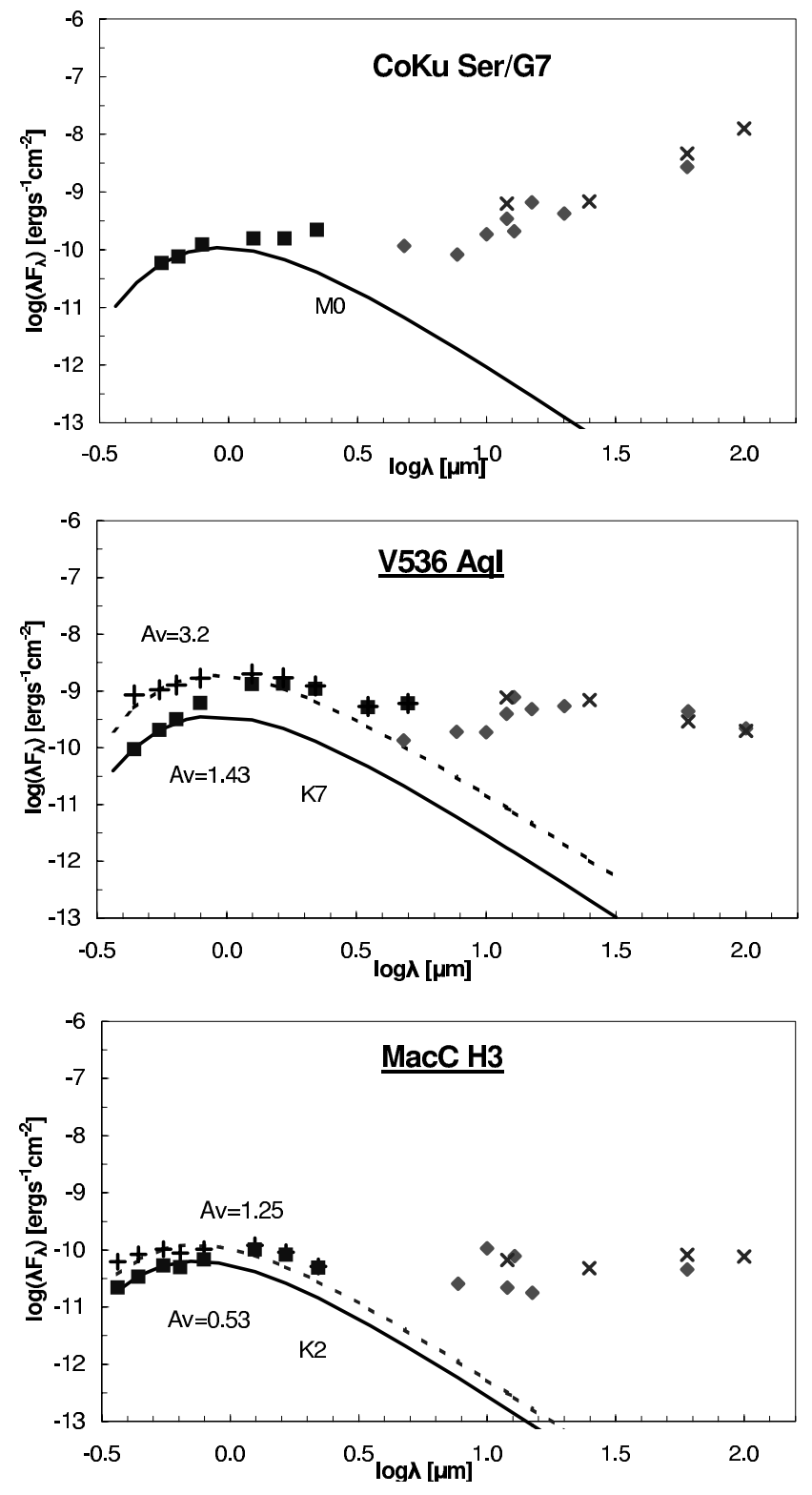

Fig. 6. SEDs (cont.) of CoKu Ser G7, V536 Aql and MacC H3, as in Fig. 1.

For those stars with companions, we have also studied, the second star's photospheric contribution to the observed fluxes, if photometry and the temperature were available in the literature.

\subsection{Notes on individual stars in the sample}

We present notes on the complete list of stars studied with comments on their spectral energy distributions, characteristics and calculations of the luminosities, infrared excesses, etc. Again we note that stars with their names underlined are CTTS.

\section{T Cha}

This star was already studied by Alcalá et al. (1993), who consider it a weak-line YY Orionis star. YY Orionis stars are TTS with strong UV emission, quick photometric and spectroscopic variability and possible mass accretion onto the star (Alcalá et al. 1993). There are ISO data presented here at more wavelengths (Fig. 1) than Alcalá et al. (1993). We have calculated

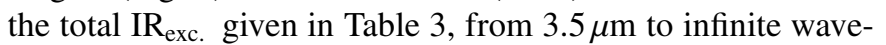
lengths, extrapolating from the longest wavelength obtained by ISO as explained earlier $(100 \mu \mathrm{m}$ for this star). In the SED (Fig. 1) of T Cha, we observed that the ISO points seem to decrease steadily from $L$ up to $20 \mu \mathrm{m}$. As the photometry at 60 and $100 \mu \mathrm{m}$ could include emission from a background source, we have also calculated the excess from $L$ to $20 \mu \mathrm{m}$, and then extrapolated the flux from $20 \mu \mathrm{m}$ to infinite wavelengths. In this latter case, the infrared excess decreases to $0.13 L_{*}\left(0.22 L_{\odot}\right)$. Alcalá et al. (1993) believe the near-infrared excess could be due to an inner accretion disk despite this being a WTTS. In fact, it has been argued (Batalha et al. 1998) that T Cha should be classified as a CTTS on the basis of the presence of this accretion from the disk.

No companion for this star has been reported in the literature.

\section{Sz 68 (HT Lup)}

This star is a WTTS and its SED (see Fig. 1) shows an excess of $0.35 L_{*}$ i.e. $1.60 L_{\odot}$, calculated from the $L$ band.

$\mathrm{Sz} 68$ has a companion at a projected separation of 390 AU $(\sim 2$. '7) with a temperature of $4000 \mathrm{~K}$ (Geoffray \& Monin 2001). Plots of both stars' photometry are shown in Fig. 7. We have calculated the extinction towards the companion using the expression from Geoffray \& Monin (2001) $A_{\mathrm{V}}=3.76 \cdot E\left(R_{\mathrm{c}}-I_{\mathrm{c}}\right)$, as we have not found photometry for the $V$ and $B$ bands. Both stars seem to have excess emission at near-infrared wavelengths, which would indicate a circumstellar disk around each, as already suggested by Geoffray \& Monin (2001). The new ISO measurements we present show quite a steeply rising infrared excess starting at $10 \mu \mathrm{m}$. In principle this excess, suggesting the presence of an extended disk, could come from either or both stars. However it seems too large to come only from $\mathrm{Sz} 68-\mathrm{B}$ (Sz $68 \mathrm{NW})$. We obtain individually calculated luminosity excesses, from $10 \mu \mathrm{m}$ onwards, of $1.36 L_{\odot}\left(0.30 L_{*, A}\right)$ for $\mathrm{Sz} 68$-A (i.e. if all the excess came from this star) and $1.51 L_{\odot}\left(11.24 L_{*, B}\right)$ if all the excess belonged to Sz68-B. We have not found any reference in the literature to the companion's $\mathrm{H} \alpha$ emission that would tell us whether the companion is a classical or a weak-line $\mathrm{T}$ Tauri star.

Sz 68-A itself is a $\sim 0{ }^{\prime} 1$ binary according to Ghez et al. (1997). This, less-luminous, third component (Sz 68-C), with a $K$-band flux ratio of 6 (Ghez et al. 1997), could be contributing to the infrared excess observed as well.

\section{$\underline{R U \text { Lup (HD142560) }}$}

Using the standard technique we were unable to calculate the extinction for RU Lup. $A_{\mathrm{V}}$ calculated from $E\left(R_{\mathrm{c}}-I_{\mathrm{c}}\right)$ yielded a negative value as well. Therefore, we used $A_{\mathrm{V}}$ from Stempels \& Piskunov (2002) instead, which was obtained from line 


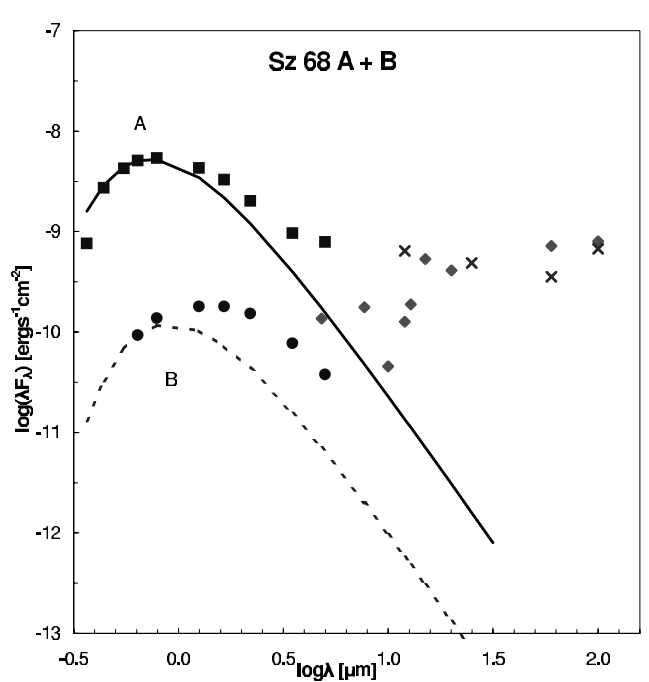

Fig. 7. SED of Sz 68 A+B. Sz 68-A is represented by a black body of $4955 \mathrm{~K}$ fitted to $R$ (continuous line). The squares are $\mathrm{U}$ to $\mathrm{L}$ band measurements of $\mathrm{Sz} 68-\mathrm{A}$, corrected for an $A_{\mathrm{V}}$ of $1.09 . \mathrm{Sz} 68-\mathrm{B}$ is represented by a black body of $4000 \mathrm{~K}$ fitted to $R$ (discontinuous line) with $A_{\mathrm{V}}=0.75$. The filled circles are the corresponding photometric measurements of Sz 68-B. The diamonds correspond to the fluxes obtained by ISO. The x's correspond to the fluxes obtained by IRAS. References for the photometry of Sz 68-A are given in Table 2. The photometry of the companion is from Hughes et al. (1994) for the $R$ and $I$ bands, and Geoffray \& Monin (2001) for $J, H, K$ and $L$.

absorption. The infrared excess from $\mathrm{H}$ to $4.8 \mu \mathrm{m}$, in Fig. 1, we thought could be due to variability and non-simultaneity of the photometry, but comparing the 2MASS-JHK photometry with photometry from Gahm et al. (1993), crosses in Fig. 1, we see there does not seem to be any variability at these wavelengths. If the $\mathrm{IR}_{\text {exc. }}$ is calculated from $4.8 \mu \mathrm{m}$, which is where the infrared excess intersects the black body curve of this star, instead of from $3.5 \mu \mathrm{m}$, it goes down from $0.68 L_{\odot}\left(0.53 L_{*}\right)$ to $0.61 L_{\odot}\left(0.48 L_{*}\right)$. 1997).

This star does not have any detected companion (Ghez et al.

\section{EX Lup (HD 325367)}

We have not been able to calculate the star's extinction, neither from $E_{B-V}$, nor from $E_{R-I}$, both giving negative $A_{\mathrm{V}}$. Furthermore, it seems to be unknown (Herbig et al. 2001). In Fig. 2, we have the photometry given by Hughes et al. (1994), filled squares, fitted by a $3802 \mathrm{~K}$ (M0.5) black body. Therefore, the luminosity of the star, uncorrected for extinction, with a spectral type M0.5, is $0.47 L_{\odot}$. The infrared excesses ratio is $0.35 L_{*}\left(0.17 L_{\odot}\right)$, calculated from $L$.

We did not find any reference to a companion for this star in the literature.

\section{Sz 124 (Th 43)}

Using a distance to the star of $125 \mathrm{pc}$ and our calculated $A_{\mathrm{V}}$ of 0.22 , we obtain a luminosity of $0.21 L_{\odot}$. The photometry from ISO shows an excess (Fig. 2) that could come from a

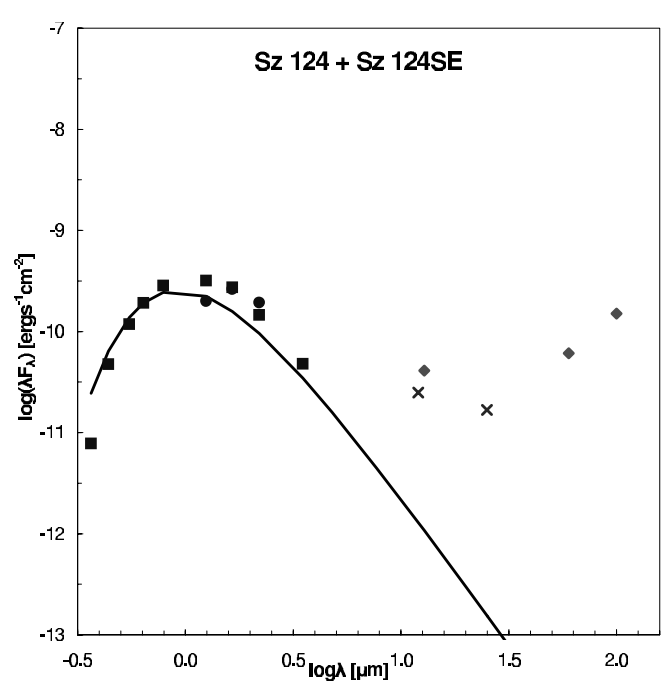

Fig. 8. SED of Sz $124+$ Sz 124SE. The black body curve of $3917 \mathrm{~K}$ belongs to $\mathrm{Sz} 124$, with the filled squares representing the star's photometry corrected for an $A_{\mathrm{V}}$ of 0.22 . Full diamonds are ISO observations and x's IRAS. The filled circles correspond to the JHK photometry given by Hughes et al. (1994) for Sz 124SE, not corrected for extinction.

circumstellar disk. The infrared excess calculated from $3.5 \mu \mathrm{m}$ is $0.81 L_{*}\left(0.17 L_{\odot}\right)$. We have also corrected the $U B V$ photometry using an $A_{\mathrm{V}}$ of 0.4 as given in Hughes et al. (1994), obtained using the $(R-I)$ color, but we have found there is not much difference in the SED, the luminosity $\left(0.25 L_{\odot}\right)$ or the infrared excess $\left(0.68 L_{*}, 0.17 L_{\odot}\right)$

Sz 124 has a companion 13". 6 away (Sz 124SE). We cannot obtain its temperature from the $J H K$ photometry, also from Hughes et al. (1994), as the extinction is unknown, but it appears (see Fig. 8) that the excess observed by ISO at $\lambda \geq$ $12.8 \mu \mathrm{m}$ could come from either or both stars. Due to the lack of information on the companion we cannot estimate its luminosity.

\section{ROX 2}

Bouvier \& Appenzeller (1992) give a spectral type for this star between $\mathrm{K} 3$ and M0. The latter spectral type is also given by Montmerle et al. (1983). In the SED graph (Fig. 2) we have plotted both the black body distribution corresponding to a K3 and an M0 star. Both black bodies seem to fit the $U B V$ photometry. It can also be seen that the $\mathrm{K} 3$ black body distribution also fits the near-infrared photometry and the flux at $12 \mu \mathrm{m}$. The extinction, however, rises from $A_{\mathrm{V}}=3.32$ (for M0) to $A_{\mathrm{V}}=5.12(\mathrm{~K} 3)$ and therefore the UBV photometry could be overdereddened. The infrared excesses found are $2.20 L_{*}$ for M0 and $0.91 L_{*}$ for $\mathrm{K} 3$, which is a $L_{\text {exc. }}$ of $2.23 L_{\odot}$, in both cases. Although we have not included it in the SED, we have also studied the case in which $A_{\mathrm{V}}=5.9$, given by Bouvier \& Appenzeller (1992) (calculated using the $V-R$ colour excess), and spectral type $\mathrm{K} 3$, but the fit to the $U B V$ photometry is very poor.

The infrared excess seems to appear at $\lambda \geq 12 \mu \mathrm{m}$. It would be possible for this emission to come from the companion 


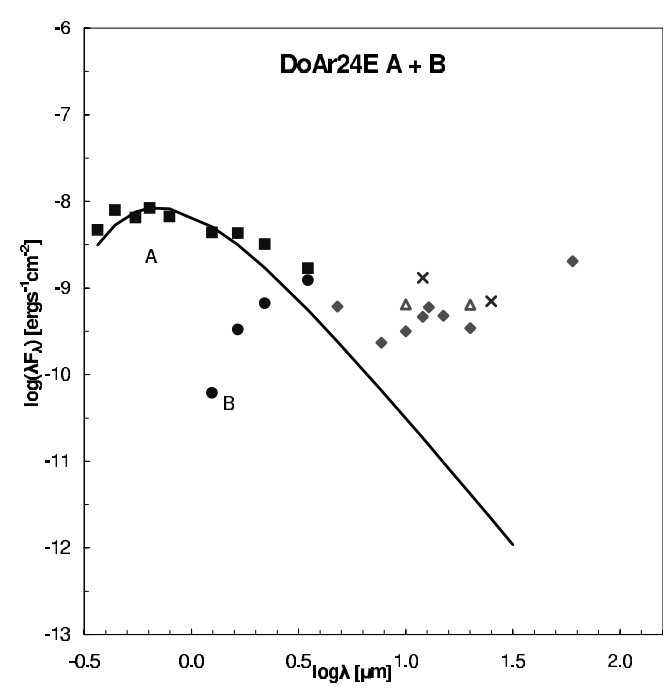

Fig. 9. SED of the system DoAr 24E A+B. The continuous line is the photosphere of DoAr 24E-A: a black body of $5236 \mathrm{~K}$ fitted to $R$; the filled squares represent the corrected photometry of the star. The filled circles are the photometry given by Chelli et al. (1988), except for J from Ageorges et al. (1997), for the companion, not corrected for extinction. Full diamonds are ISO measurements. Crosses are IRAS measurements. Empty triangles are photometry at 10 and $20 \mu \mathrm{m}$, from Chelli et al. (1988).

found 0.'41 away by Barsony et al. (2003) and references therein. This companion would have a flux at the $K$ band of $0.57 K_{\mathrm{ROX} 2}$ Barsony et al. (2003).

\section{DoAr 24 (ROX 10A)}

From Bouvier \& Appenzeller (1992) we have a spectral type of K5. There is an accuracy of \pm 2 subclasses, so we have also included in Fig. 3, the black body of a K3 star, which fits the near-infrared photometry better. There is a corresponding decrease in the infrared excess from $2.17 L_{*}\left(3.42 L_{\odot}\right)$ to $1.48 L_{*}\left(2.34 L_{\odot}\right)$. If the extrapolation to infinite wavelength is done from $20 \mu \mathrm{m}$, the excess decreases even more (to $0.05 L_{*}$, $0.12 L_{\odot}$, for the $\mathrm{K} 3$ spectral type).

No companion is reported in the literature and the infrared emission seems to indicate the presence of a circumstellar disk.

\section{DoAr 24E (ROX 10B)}

Using the spectral type of K0 given by Bouvier \& Appenzeller (1992) we obtain an extinction of $A_{\mathrm{V}}=5.84$, with $R_{V}=4.2$ (filled squares and continuous line in Fig. 3). If we use the extinction of $A_{\mathrm{V}}=6.6$, also from Bouvier \& Appenzeller (1992) and calculated from the $(V-R)$ colour excess, the black body passes through the 4.8 and $10 \mu \mathrm{m}$ ISO points but $U$ and $B$ appear to be over-corrected (crosses and discontinuous line in Fig. 3). We obtain an excess of $0.47 L_{*}\left(3.97 L_{\odot}\right)$ in the first case and $0.22 L_{*}\left(3.82 L_{\odot}\right)$ in the second. If we do not include the flux observed at $60 \mu \mathrm{m}$ and extrapolate from the flux at $20 \mu \mathrm{m}$ the excess decreases to $0.08 L_{*}\left(0.68 L_{\odot}\right)$.

Chelli et al. (1988) suggest the infrared excess from $L$ onwards is emitted by an infrared companion at $\sim 2$.! 3 . They find

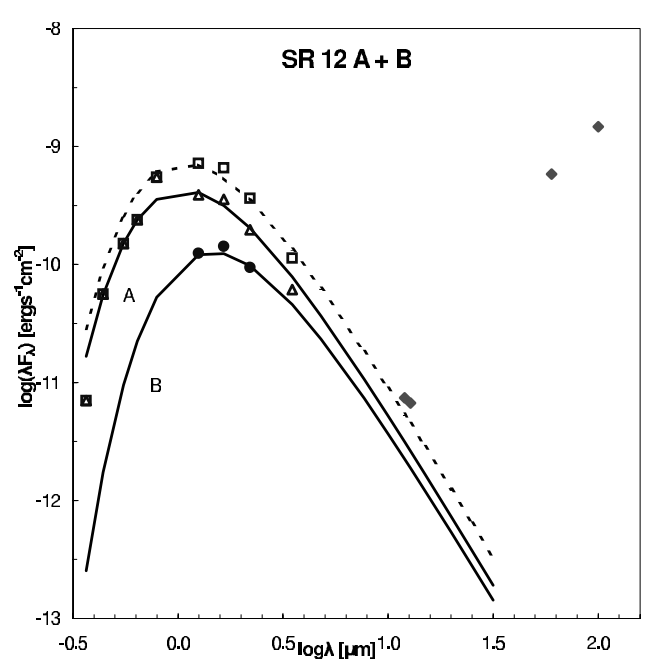

Fig. 10. SED of the system SR 12 A+B. A is the photosphere of SR 12A with the empty triangles representing the corrected photometry of the star. $B$ is the photosphere of SR 12-B with the filled circles being the apparent JHKL photometry of the companion. The empty squares are the photometry observed for the whole system. The discontinuous line corresponds to the sum of the blackbody fits for the two stars. The diamonds correspond to the fluxes obtained with ISO. References for the photometry are given in Table 2, except for SR12-B and the whole system which are from Simon et al. (1987).

$L_{*}($ DoAr 24E-A $)=7.9 \quad L_{\odot} \quad$ and $\quad L_{*}($ DoAr 24E-B $)=1.4 \quad L_{\odot}$, assuming all the flux at $\lambda \leq 3.5 \mu \mathrm{m}$ to come from the main star and the rest from the companion. In Fig. 9 we have plotted (filled dots) the photometry of the companion (Chelli et al. 1988), not corrected for extinction, alongside the SED of the main star. 2MASS photometry for the companion does not seem to be available. Although the presence of a companion and its contribution to the observed photometry cannot be ignored, the dip in the new fluxes observed by ISO and the increase in the infrared emission at longer wavelengths, seems to indicate the presence of a disk.

\section{SR 12 (ROX 21, V2247 Oph)}

Bouvier \& Appenzeller (1992) state a slightly higher value than Cohen \& Kuhi (1979), for the $E W(\mathrm{H} \alpha)$. It is still, however, less than $10 \AA$, and therefore according to the standard definition a WTTS. The most recent paper (Bouvier \& Appenzeller 1992) states the spectral type to be between K4 and M2.5. Previous papers give spectral types of M1 (Rydgren et al. 1976; Cohen \& Kuhi 1979) and M2.5 (Rydgren 1980). In the SED graph (Fig. 3) we have included both the black body distribution corresponding to a $\mathrm{K} 4$ star and a M2.5 star. The K4 black body fits the near-infrared photometry, and the flux observed at $12 \mu \mathrm{m}$, better. The extinction, however, rises to $A_{\mathrm{V}}=2.69$, from 0.46 for M2.5. The infrared excess found if a spectral type of M2.5 is assumed is $2.65 L_{*}\left(2.4 L_{\odot}\right)$. If $\mathrm{K} 4$ were to be adopted, there would still be a significant infrared excess of $1.18 L_{*}\left(2.4 L_{\odot}\right)$.

Simon et al. (1987) found SR 12 to be a $\sim 0^{\prime} 30$ binary star ( $\sim 50 \mathrm{AU})$, from $K$ band lunar occultation observations. In their paper, they present $K$ band magnitudes for both components and flux ratios for the $J$ and $H$ magnitudes. They suggest 


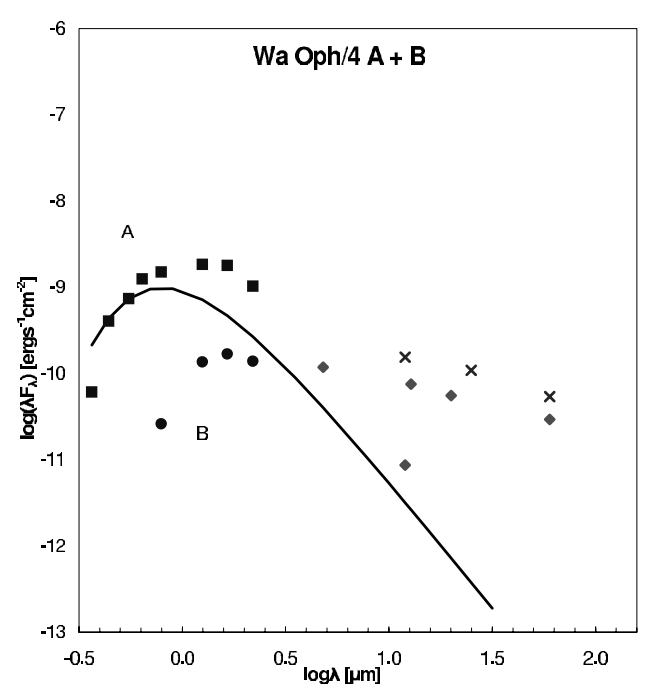

Fig. 11. SED of Wa Oph/4 A and B. The continuous line is the photosphere of Wa Oph/4-A: a K4 black body $(4500 \mathrm{~K})$ fitted to $V$; the filled squares represent the corrected photometry for Wa Oph/4-A. The filled circles are the uncorrected photometry of the companion, Wa Oph/4-B. The diamonds correspond to the fluxes obtained by ISO. The x's correspond to the fluxes obtained by IRAS for Wa Oph/4. References for the photometry of the primary are given in Table 2. The photometry of the companion is from DENIS ( $I$-band) and 2MASS $(J$, $H$ and $K$ bands).

temperatures of $5000 \mathrm{~K}$ for SR $12-\mathrm{A}$ and $2500 \mathrm{~K}$ for SR 12 -B. Although a black body with effective temperature of $5000 \mathrm{~K}$ would fit the infrared photometry, we find the spectral type of M2.5 (3428 K) to be better for all the photometry available (from $\mathrm{U}$ to $\mathrm{L}$ ). In Fig. 10, we have plotted the black body curves (normalized to go through K) corresponding to SR 12-A $(3428 \mathrm{~K})$ and SR $12-\mathrm{B}(2500 \mathrm{~K})$. It can be seen that the total infrared flux (at $J, H$ and $K$ ) of the two stars, is approximately fitted by the sum of the two black body curves (discontinuous line in Fig. 10), as well as the flux obtained with ISO at $12 \mu \mathrm{m}$. There is still a clear infrared excess but only at the longest ISO wavelengths.

If we suppose the infrared emission to come from a circumbinary disk, we obtain the disk luminosity $1.71 L_{\text {binary }}$ (with $L_{\text {binary }}$ being the sum of the luminosities of the two stars). No other companion has been reported nearby but we believe there must be an embedded infrared companion present to explain the large infrared excess we find.

\section{ROX 31}

For this star we have found no data corresponding to $3.5 \mu \mathrm{m}$, so the infrared excess has been calculated from $4.8 \mu \mathrm{m}$. Using $\mathrm{K} 7$ from Bouvier \& Appenzeller (1992), we obtain the same $A_{\mathrm{V}}$ (4.2) and $L_{*}\left(0.48 L_{\odot}\right)$ given in their paper. Taking into account the \pm 2 subclass error in the spectral type we found that even using $\mathrm{K} 5$ or M1 as the star's temperature, the $H$ and $K$ points were not fitted by a black body. The only possibility was to make $A_{\mathrm{V}}=5.5$, maintaining $\mathrm{K} 7$. In this case the black body passes through $H, K$ and the flux observed at $4.8 \mu \mathrm{m}$. Then the infrared excess is $0.33 L_{*}\left(0.52 L_{\odot}\right)$, instead of the $1.15 L_{*}$

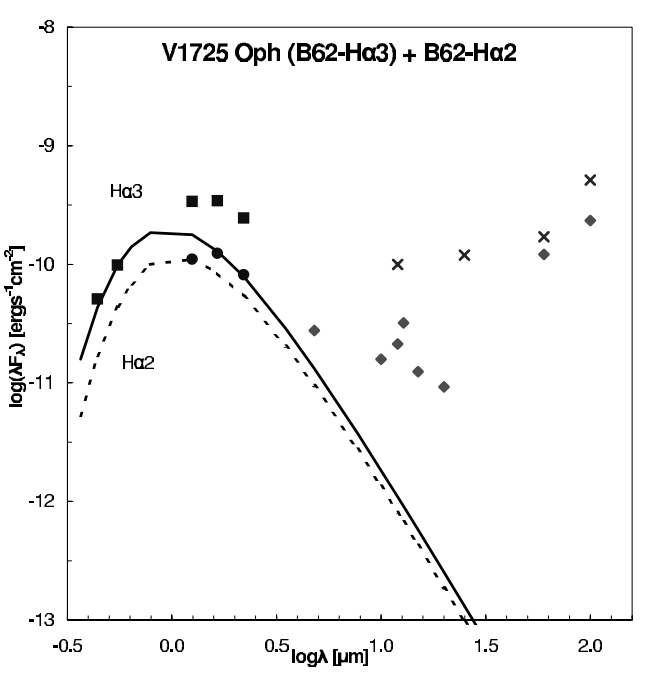

Fig. 12. SED of V1725 Oph ( $\mathrm{H} \alpha 3)$ and $\mathrm{B} 62-\mathrm{H} \alpha 2(\mathrm{H} \alpha 2)$. The continuous line is the photosphere of V1725 Oph: a black body of $3802 \mathrm{~K}$ (M0.5) fitted to $V$; the filled squares represent the corrected photometry of the star. The discontinuous line is the photosphere of $\mathrm{B} 62-\mathrm{H} \alpha 2$ : a black body of $3500 \mathrm{~K}$ fitted to $J$, with the filled circles being the uncorrected photometry of this second star. The diamonds correspond to the fluxes obtained by ISO. The x's correspond to the fluxes obtained by IRAS for B62-H $\alpha 2$. These fluxes could also come from V1725 Oph (Weaver \& Jones 1992). References for the photometry of V1725 Oph are given in Table 2. The photometry of the nearby star is from Reipurth \& Gee (1986).

value $\left(0.55 L_{\odot}\right)$ found with $A_{\mathrm{V}}=4.2$. We have also included this option in Fig. 4, although supposing $A_{\mathrm{V}}=5.5$ seems to overcorrect the $U B V$ magnitudes.

Simon et al. (1987) observed a companion at a projected angular separation of $\sim 130^{\prime \prime}$. This companion should not contribute to the infrared emission observed, but Barsony et al. (2003), and references therein, reported this star to have a second companion 0 ' 39 away. The magnitude difference in the $K$ band between ROX 31 and this second companion is very small $\left(\delta m_{K}=0.3\right.$, according to Ageorges et al. 1997). It is difficult to say whether the large $\mathrm{IR}_{\text {exc. }}$ comes only from a circumstellar disk or also from the subarcsecond companion.

\section{Wa Oph/4 (V2507 Oph)}

Walter (1986) assumes $R_{V}$ to be 3.1. We have also included in the graph (Fig. 4) the black body if $R_{V}=4.2$. This seems to bring the black body closer to fitting the 4.8 and $12 \mu \mathrm{m}$ points and also fits the near-infrared photometry. If $R_{V}=4.2$, which gives an $A_{\mathrm{V}}$ nearer to the value found in Chavarría (2000) obtained from Strömgren photometry, the infrared excess would be $0.04 L_{*}\left(0.10 L_{\odot}\right)$, i.e. there would be less than half as much infrared excess, but still enough to suggest the presence of a disk.

According to Reipurth \& Zinnecker (1993), Wa Oph/4 has a companion $\sim 88^{\prime \prime} 7$ away. We have plotted both stars' photometry in Fig. 11. Jensen et al. (2004) detected and measured polarized light from both stars (the polarization angle tends to be either parallel or orthogonal to the disk rotation axis). Hence, they imply both stars in the system have disks, confirming the 


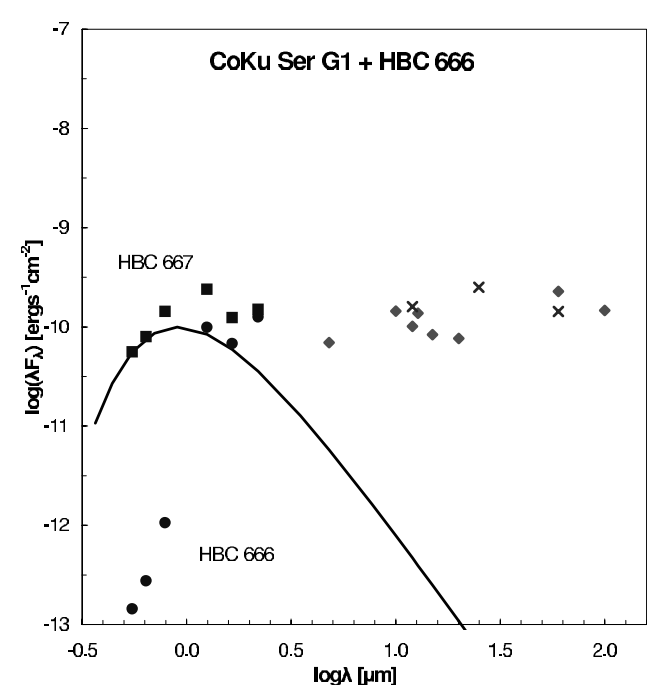

Fig. 13. SED of the system CoKu Ser G1 (HBC 667) and HBC 666. The black body curve belongs to CoKu Ser G1, with the filled squares representing the star's photometry (see references in Table 2) corrected for an $A_{\mathrm{V}}$ of 3.48. Full diamonds are ISO observations and x's IRAS. The filled circles correspond to the photometry of the companion HBC 666, not corrected for extinction, with $V$ (20.4 mag), $R$ $(19.3 \mathrm{mag})$ and $I(17.4 \mathrm{mag})$ from the observations taken in La Palma for this paper and $J, H$ and $K$ from 2MASS.

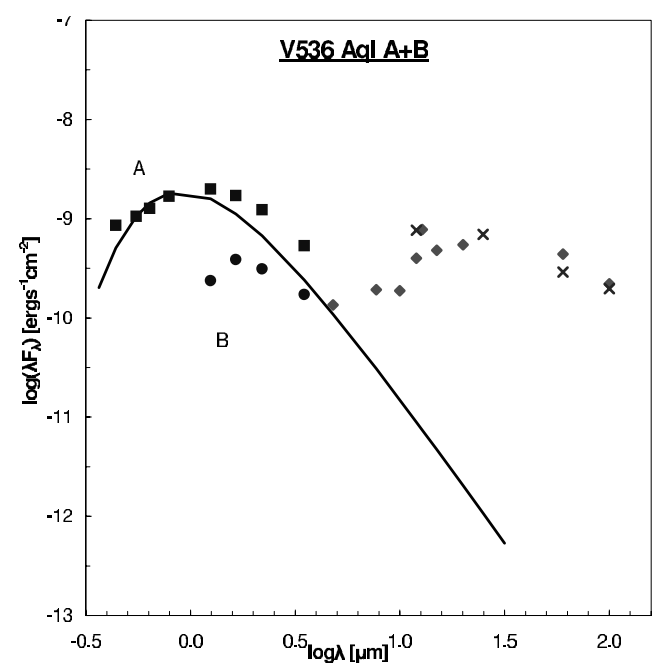

Fig. 14. SED of the system V536 Aql A+B. A is the photosphere of V536 Aql-A: the squares represent the corrected photometry and the continuous line is a black body of $4000 \mathrm{~K}$ fitted to $\mathrm{V}$. The filled circles are the uncorrected $J H K L$ photometry of V536 Aql-B. The diamonds correspond to the fluxes obtained by ISO. The $x$ 's correspond to the fluxes obtained by IRAS. References for the photometry of V536 AqlA are given in Table 2. The photometry of the companion is from Geoffray \& Monin (2001).

interpretation that the infrared excess seen for Wa Oph/4 comes from a disk around the WTTS (although the infrared excess at longer wavelengths seen in Fig. 11 could include a contribution from both disks).

\section{Wa Oph/6 (V2508 Oph)}

In the graph (Fig. 4), we have the corrected available $U$ to $K$ magnitudes and black body curve where: $1 . A_{\mathrm{V}}=1.24$, calculated using $R_{V}=3.1 ; 2$. $A_{\mathrm{V}}=1.68$, calculated using $R_{V}=4.2$; 3. $A_{\mathrm{V}}=2.74$, calculated by Chavarría (2000), from their Strömgren photometry. The infrared excess decreases from $0.96 L_{*}\left(0.64 L_{\odot}\right)$, to $0.23 L_{*}\left(0.62 L_{\odot}\right)$, for cases 1 to 3 . The excess has been calculated from $4.8 \mu \mathrm{m}$ although it seems to start around $1.65 \mu \mathrm{m}$. Photometry in the $J H K$ bands appear to be better fitted by a black body normalized to $V$ and corrected for $A_{\mathrm{V}}=2.74$.

This star does not seem to have a companion and the infrared excess probably comes from a circumstellar disk, in line with it being a CTTS.

\section{Oph}

No more information about the star's spectral type was found apart from the fact that it is of $\mathrm{G}$ type (Covino et al. 1992). This star does not have any infrared excess detected by ISO (Fig. 5). We note that it has one of the lowest $\mathrm{H} \alpha$ equivalent widths in Table 3.

\section{V1725 Oph (B62-H $\alpha 3)$}

Reipurth \& Gee (1986) present a range of values for $B$ and $V$. In the SED (Fig. 5), we have plotted the averages corrected for extinction using our calculated $A_{\mathrm{V}}=0.28$ (crosses) and $A_{\mathrm{V}}=1.08$ (filled squares), obtained from Strömgren photometry by Reipurth \& Gee (1986), both fitted with black body distributions for an M0.5 star. It can be seen that if an extinction of $A_{\mathrm{V}}=1.08$ is used, the curve is closer to the $4.8 \mu \mathrm{m}$ point. The luminosity of the star and the infrared excess, have been calculated using the black body curve fitted to the average magnitudes with $A_{\mathrm{V}}=1.08$ (solid-line curve in the graph).

This WTTS has not been reported to be a binary star but Weaver \& Jones (1992) noted that the IRAS emission associated with HBC 659 (B62-H $\alpha 2$ ), 30" away, could be from V1725 Oph as well. We have plotted the SEDs of both stars with the ISO and IRAS fluxes in Fig. 12. The infrared emission observed by ISO at $\lambda \geq 20 \mu \mathrm{m}$ could be including emission from a disk around B62-H $\alpha 2$. Reipurth \& Gee (1986) give a spectral type for $\mathrm{B} 62-\mathrm{H} \alpha 2$ of $\mathrm{M} 2$ and an $E W(\mathrm{H} \alpha)$ of $19 \AA$ (it is considered to be a classical T Tauri star). We find a luminosity for this star of $0.43 L_{\odot}$.

\section{CoKu Ser G1}

For CoKuSer G1, we have used $A_{\mathrm{V}}=3.48$, Cohen \& Kuhi (1979), determined from image-tube-scanner narrow band indices between $\left(0.54 \mu \mathrm{m}-R_{\mathrm{c}}\right)$ and $\left(0.60 \mu \mathrm{m}-R_{\mathrm{c}}\right)$, with $R=3.1$ for the reddening law, as we do not have the star's $B$ magnitude. The second black body present in the graph (Fig. 5) corresponds to $A_{\mathrm{V}}=4.02$, calculated using $A_{\mathrm{V}}=3.76 \cdot E\left(R_{\mathrm{c}}-I_{\mathrm{c}}\right)$, Geoffray \& Monin (2001), which seems to fit the near-infrared photometry slightly better. In the second case, the luminosity of the star increases from $0.60 L_{\odot}$ to $0.99 L_{\odot}$ and the infrared excess decreases from $3.70 L_{*}\left(2.21 L_{\odot}\right)$ to $2.24 L_{*}\left(2.21 L_{\odot}\right)$. 
Table 3. $\mathrm{H} \alpha$ equivalent widths, T Tauri classification, spectral types, effective temperatures and distances of the stars studied as found in the literature and used to calculate the stellar luminosities and infrared excesses.

\begin{tabular}{|c|c|c|c|c|c|c|c|c|c|c|c|c|c|c|c|c|}
\hline \multirow[t]{2}{*}{ Star } & \multirow{2}{*}{$\begin{array}{c}E W(\mathrm{H} \alpha) \\
{[\AA]}\end{array}$} & \multirow[t]{2}{*}{ TTS } & \multirow[t]{2}{*}{ Ref. } & \multirow[t]{2}{*}{ Sp. T. } & \multirow[t]{2}{*}{ Ref. } & \multirow{2}{*}{$\begin{array}{c}T \\
{[\mathrm{~K}]}\end{array}$} & \multirow[t]{2}{*}{$A_{\mathrm{V}}$} & \multirow[t]{2}{*}{ Ref. } & \multirow{2}{*}{$\begin{array}{c}d \\
{[\mathrm{pc}]}\end{array}$} & \multirow[t]{2}{*}{ Ref. } & \multirow{2}{*}{$\begin{array}{c}L_{*} \\
{\left[L_{\odot}\right]}\end{array}$} & \multirow{2}{*}{$\begin{array}{c}\lambda_{\mathrm{IR}}{ }^{a} \\
{[\mu \mathrm{m}]}\end{array}$} & \multirow{2}{*}{$\begin{array}{l}L_{\text {exc. }} \\
{\left[L_{\odot}\right]}\end{array}$} & \multicolumn{2}{|c|}{$\mathrm{IR}_{\text {exc. }}{ }^{b}$} & \multirow[t]{2}{*}{ co. ${ }^{c}$} \\
\hline & & & & & & & & & & & & & & o & 1 & \\
\hline T Cha & - & $\mathrm{w}$ & 1 & G8 & 1 & 5445 & 1.27 & $\mathrm{C}$ & 140 & 2 & 1.71 & 3.5 & 0.63 & 0.36 & 0.13 & $\mathrm{n}$ \\
\hline Sz 68 & $2-7$ & W & 3 & K2 & $3 / 4$ & 4955 & 1.09 & C & 150 & 3 & 4.59 & 3.5 & 1.60 & 0.35 & 0.30 & $\mathrm{y}$ \\
\hline RU Lup & 216.4 & $\mathrm{c}$ & 5 & K7-M0 & 6 & 3963 & 0.20 & 6 & 127 & 7 & 1.27 & 3.5 & 0.68 & 0.53 & 0.48 & $\mathrm{n}$ \\
\hline EX Lup & 43.3 & $\mathrm{c}$ & 5 & M0.5 & 5 & 3802 & $?$ & 18 & 125 & 5 & 0.47 & 3.5 & 0.17 & 0.35 & - & $\mathrm{n}$ \\
\hline Sz 124 & 1.6 & $\mathrm{w}$ & 5 & M0 & 5 & 3917 & 0.22 & $\mathrm{C}$ & 125 & 5 & 0.21 & 3.5 & 0.17 & 0.81 & - & $\mathrm{y}$ \\
\hline ROX 2 & 2.8 & $\mathrm{w}$ & 8 & M0 & 8 & 3917 & 3.32 & $\mathrm{C}$ & 170 & 8 & 1.01 & 12.0 & 2.23 & 2.20 & 0.91 & $\mathrm{y}$ \\
\hline DoAr 24 & 20.0 & $\mathrm{c}$ & 8 & K5 & 8 & 4395 & 3.15 & $\mathrm{C}$ & 170 & 8 & 1.58 & 4.8 & 3.42 & 2.17 & 0.05 & $\mathrm{n}$ \\
\hline DoAr 24E & 5.0 & $\mathrm{w}$ & 8 & K0 & 8 & 5236 & 5.84 & $\mathrm{C}$ & 170 & 8 & 8.50 & 3.5 & 3.97 & 0.47 & 0.08 & $\mathrm{y}$ \\
\hline SR 12 & 3.6 & $\mathrm{w}$ & 9 & M2.5 & 10 & 3428 & 0.46 & $\mathrm{C}$ & 170 & 8 & 0.91 & 12.0 & 2.41 & 2.65 & $1.71^{s}$ & $\mathrm{y}$ \\
\hline ROX 31 & 3.3 & $\mathrm{w}$ & 8 & K7 & 8 & 4000 & 4.20 & $\mathrm{C}$ & 170 & 8 & 0.48 & 4.8 & 0.55 & 1.15 & 0.33 & $\mathrm{y}$ \\
\hline $\mathrm{Wa} \mathrm{Oph/4}$ & 0.6 & $\mathrm{w}$ & 11 & K4 & 11 & 4581 & 2.40 & $\mathrm{C}$ & 150 & 11 & 1.00 & 4.8 & 0.11 & 0.11 & 0.04 & $\mathrm{y}$ \\
\hline Wa Oph/6 & 35.0 & $\mathrm{c}$ & 11 & K7 & 12 & 4000 & 1.24 & $\mathrm{C}$ & 150 & 11 & 0.67 & 4.8 & 0.64 & 0.96 & 0.23 & $\mathrm{n}$ \\
\hline IX Oph & 1.3 & $\mathrm{w}$ & 9 & $\mathrm{G}$ & 13 & 5902 & 2.29 & $\mathrm{C}$ & 125 & 14 & 2.80 & 3.5 & 0.06 & 0.02 & - & $\mathrm{n}$ \\
\hline V1725 Oph & 6.5 & $\mathrm{w}$ & 15 & M0.5 & 15 & 3802 & 1.08 & 15 & 225 & 15 & 0.63 & 4.8 & 0.86 & 1.37 & - & $\mathrm{n}$ ? \\
\hline CoKu Ser G1 & 3.3 & $\mathrm{w}$ & 9 & K7 & 9 & 4000 & 3.48 & 9 & 350 & 16 & 0.60 & 4.8 & 2.21 & 3.70 & 2.24 & $\mathrm{y}$ \\
\hline CoKu Ser G7 & 5.5 & $\mathrm{w}$ & 9 & M0 & 9 & 3917 & 3.72 & 9 & 350 & 16 & 0.82 & 4.8 & 20.09 & 24.52 & 0.65 & $\mathrm{n}$ ? \\
\hline V536 Aql & 51.8 & $\mathrm{c}$ & $9 / 3$ & K7 & 3 & 4000 & 1.43 & $\mathrm{C}$ & 200 & 3 & 0.72 & 4.8 & 1.79 & 2.48 & 0.47 & $\mathrm{y}$ \\
\hline $\mathrm{MacC} \mathrm{H} 3$ & 11.4 & $\mathrm{c}$ & 9 & $\mathrm{~K} 2$ & 4 & 4955 & 0.53 & $\mathrm{C}$ & 845 & 17 & 1.83 & 7.7 & 3.03 & 1.66 & 0.85 & $\mathrm{n}$ \\
\hline
\end{tabular}

Notes to Table: "C" in the extinction's reference column indicates we have calculated it for this paper from the photometry and the spectral type. Otherwise the reference is given.

${ }^{a}$ Wavelength (in $\mu \mathrm{m}$ ) from where the infrared luminosity excess was calculated (to infinite wavelength);

${ }^{b} \mathrm{IR}_{\text {exc. }}=\left(L_{\mathrm{IR}}-L_{\mathrm{bb}}\right) / L_{*}$; (o) are the excesses calculated over the continuous line representing the star, seen in each graph (Figs. 1 to 6); (1) are the lower limits obtained for the excesses. "s" indicates the infrared excess is over the luminosity of the binary system. Details on how each infrared excess for individual stars was obtained, can be found in Sect. 3;

${ }^{c}$ If the star is believed to have a companion nearby (y) or not (n), according to the literature. "n?" no companion has been reported but we believe there could be one.

References: (1) Alcalá et al. (1993), (2) Siebenmorgen et al. (2000), (3) Geoffray \& Monin (2001), (4) Herbig-Bell Catalogue (HBC), (5) Appenzeller et al. (1983), (6) Stempels \& Piskunov (2002), (7) Favata et al. (1998), (8) Bouvier \& Appenzeller (1992), (9) Cohen \& Kuhi (1979), (10) Rydgren et al. (1976), (11) Walter (1986), (12) Chavarría (2000), (13) Covino et al. (1992), (14) Reipurth et al. (1996), (15) Reipurth \& Gee (1986), (16) Brown et al. (2000), (17) de Zeeuw et al. (1999), (18) Herbig et al. (2001).

$\mathrm{CoKu}$ Ser G1 is a 3.'5 pre-main-sequence binary (Jensen et al. 1996). The companion (HBC 666) has an $E W(\mathrm{H} \alpha)=14.4 \AA$ (Cohen \& Kuhi 1979), but no spectral type is given. We have also obtained from La Palma photometry for the companion in the $V, R$ and $I$ bands (see Fig. 13). The IRAS fluxes shown in Fig. 13 may include contributions from HBC 666, CoKu Ser G1 and even CoKu Ser G2, another CTTS less than 30" away (Weaver \& Jones 1992). Although the presence of a disk is clearly inferred, it is difficult to say to which stars it belongs to.

\section{CoKu Ser G7}

For this star we have also used $A_{\mathrm{V}}$ from Cohen \& Kuhi (1979) and we can see in Fig. 6, the infrared excess seems to start from $\mathrm{H}$. The excess calculated from $4.8 \mu \mathrm{m}$ appears to be large.

$\mathrm{CoKu}$ Ser G7 is situated in the middle of the Serpens Cloud Core and 2MASS detected several additional infrared sources at distances $\leq 10^{\prime \prime}$, although no companion for this star has been reported in the literature. Assuming a large background contribution at 60 and $100 \mu \mathrm{m}$, we have conservatively calculated the infrared excess up to $20 \mu \mathrm{m}$ and extrapolate from this wavelength, obtaining an $\mathrm{IR}_{\text {exc. }}$ of $4.53 L_{*}\left(3.71 L_{\odot}\right)$. Furthermore, if only the shorter wavelengths infrared excess (up to the $7.7 \mu \mathrm{m}$ point) were from the circumstellar disk, the $\mathrm{IR}_{\text {exc. would be } 0.65 L_{*}}\left(0.54 L_{\odot}\right)$.

\section{V536 Aql}

We have calculated $A_{\mathrm{V}}$ for this star from the photometry taken in La Palma. Using this $A_{\mathrm{V}}=1.43$, we obtain a luminosity of $0.72 L_{\odot}$ and we observe (Fig. 6) an infrared excess which starts after $R$ and has a dip around the $7.7 \mu \mathrm{m}$ point. We have also plotted the black body fit to the photometry now corrected for an $A_{\mathrm{V}}=3.2$ from Cohen \& Kuhi (1979), calculated as mentioned earlier, that seems to go through $B$ up to $4.8 \mu \mathrm{m}$. With this second extinction, the luminosity of the star goes up from $0.72 L_{\odot}$ to $3.69 L_{\odot}$, and the infrared excess decreases from $2.48 L_{*}\left(1.79 L_{\odot}\right)$ to $0.47 L_{*}\left(1.75 L_{\odot}\right)$.

V536 Aql is a binary star with the companion estimated to be 0.'52 away ( $\sim 100 \mathrm{AU})$, Ageorges et al. (1994). In Fig. 14 we have plotted both stars' fluxes using JHKL for V536 Aql-B from Geoffray \& Monin (2001). The companion could also 


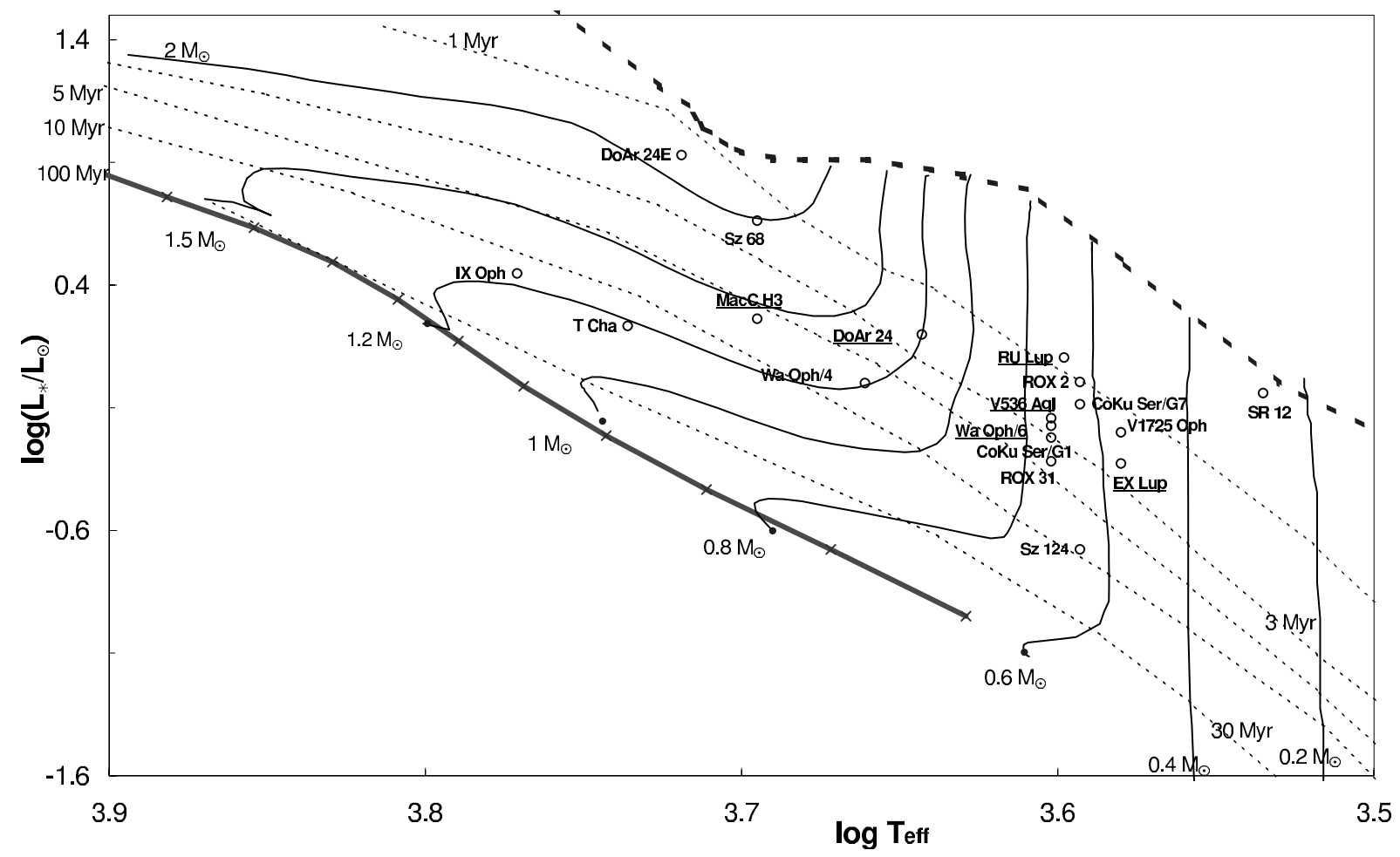

Fig. 15. H-R diagram with evolutionary tracks to estimate the masses and ages of the stars observed. Stars with their names underlined are CTTS. The others are WTTS. The ZAMS (thick continuous line) is from VandenBerg \& Bridges (1984). The evolutionary tracks (thin continuous lines), isochrones (thin discontinuous lines) and birthline (thick discontinuous line) are from Palla \& Stahler (1999).

have a disk and its infrared emission be included in the excess observed for V536 Aql.

Hirth et al. (1997) and Mundt \& Eislöffel (1998) observed outflows from this star, implying the clear presence of an accretion disk.

\section{$\underline{\mathrm{MacC} \mathrm{H3}}$}

Cohen \& Kuhi (1979) give a H $\alpha$ equivalent width of $11.4 \AA$, and Fernández et al. (1995) of $23.7 \AA$. It is therefore a CTTS. Besides the black body appropriate to the calculated extinction $\left(A_{\mathrm{V}}=0.53\right)$, we have included the corresponding black body if $A_{\mathrm{V}}=1.25$ (Cohen \& Kuhi 1979) in Fig. 6 ( $A_{\mathrm{V}}$ obtained as explained in the notes for CoKu Ser G1). If the latter extinction is the correct one, the luminosity of the star increases to $3.56 L_{\odot}$ (from $1.83 L_{\odot}$ ) and the infrared excess decreases from $1.66 L_{*}$ to $0.85 L_{*}$, with an $L_{\text {exc. }}$ in both cases of $3.03 L_{\odot}$.

\section{Discussion}

From the spectral energy distributions of the 12 weak-line $\mathrm{T}$ Tauri stars studied and the results summarized in Table 3 (for the main stars) and Table 4 (for the companions), we see that:

- T Cha has a probable circumstellar disk and has not been reported to be a binary system.

- Sz 68, Sz 124, ROX31, DoAr 24E, Wa Oph/4, V1725 Oph and CoKu Ser G1 could have both circumstellar disks and companions. Furthermore, the companions of the last two stars are known to be CTTS.
- CoKu Ser G7 seems deeply embedded in the molecular cloud and the origin of the IR emission at longer wavelengths is unclear but a circumstellar disk can be inferred if only the emission up to $20 \mu \mathrm{m}$ is considered to come from the star itself.

- IX Oph appears devoid of any infrared excess. This star is also the one nearest to the ZAMS, and may be a postT Tauri star.

- SR 12's infrared excess seems to come from its companion (or a nearby infrared source). Whether there are disks around them or not, remains uncertain. This could also be the case with ROX 2, as it has an SED similar to SR 12's and a companion. We note however that, Littlefair et al. (2004), suggest SR 12 and ROX 2 are WTTS with accretion disks. They compared new spectra with composite-spectra of the same stars taken 20 years ago and have found an increase in $E W(\mathrm{H} \alpha)$. They argue the change in $E W(\mathrm{H} \alpha)$ can be explained by the variation in the properties of an accretion disk that must be present, in spite of their lack of near-infrared excess.

Half of the SEDs of the WTTS with excess show a flux deficit at 7.7 or $10 \mu \mathrm{m}$ suggesting the presence of a dust free hole in the inner portion of the disk (Calvet et al. 2002).

On the other hand, all 6 CTTS studied have infrared excesses that seem to indicate the presence of circumstellar disks. Of these, 4 also show dips in the SEDs at $10 \mu \mathrm{m}$, as in the case of the WTTS. 
Table 4. List of known secondaries to the T Tauri stars in our sample.

\begin{tabular}{lccccccc}
\hline \hline Star & $\begin{array}{c}E W(\mathrm{H} \alpha)^{a} \\
{[\AA]}\end{array}$ & $\begin{array}{c}T^{a} \\
{[\mathrm{~K}]}\end{array}$ & $A_{\mathrm{V}}{ }^{a}$ & $\begin{array}{c}L_{*}{ }^{b} \\
{\left[L_{\odot}\right]}\end{array}$ & $\begin{array}{c}L_{\text {exc. }}{ }^{c} \\
{\left[L_{\odot}\right]}\end{array}$ & $\begin{array}{c}\mathrm{IR}_{\text {exc. }}{ }^{c} \\
{\left[L_{*}\right]}\end{array}$ & $\begin{array}{c}d^{d} \\
{\left[{ }^{\prime \prime}\right]}\end{array}$ \\
\hline Sz 68-B & - & 4000 & 0.75 & 0.13 & 1.5 & 11.2 & 2.7 \\
Sz 124SE & - & - & - & - & - & - & 13.6 \\
ROX 2-B & - & - & - & - & - & - & 0.41 \\
DoAr 24E-B & - & - & - & 1.40 & - & - & 2.3 \\
SR 12B & - & 2500 & - & 0.50 & 2.4 & 4.8 & 0.3 \\
ROX 31-B & - & - & - & - & - & - & 0.39 \\
Wa Oph/4-B & - & - & - & - & - & - & 8.7 \\
B62-H $\alpha 22^{e}$ & 19 & 3500 & - & 0.43 & - & - & 30 \\
HBC 666 & 14 & - & - & - & - & - & 3.5 \\
V536 Aql-B & - & - & - & - & - & - & 0.52 \\
\hline
\end{tabular}

Notes to table:

${ }^{a}$ References for the $\mathrm{H} \alpha$ equivalent width, temperature and extinction can be found in the corresponding entry for each star in Sect. 3 .

${ }^{b}$ The stellar luminosities were obtained from the corresponding black body curves fitted to the available photometry for each star, except for DoAr24E-B, which is from Chelli et al. (1988). Av was taken to be zero unless otherwise stated.

${ }^{c}$ The infrared luminosity excess $\left(L_{\text {exc. }}\right)$ and $\mathrm{IR}_{\text {exc. }}$ have been calculated over the same range as the main star.

${ }^{d} d$ is the angular distance from the companion to the primary.

${ }^{e}$ This star is not really a companion but a star near V1725 Oph, associated with the same globule Barnard 62 (Reipurth \& Gee 1986; Weaver \& Jones 1992).

${ }^{f}$ Companion of CoKu Ser/G1, also known as CoKu Ser/G1/c (Cohen \& Kuhi 1979).

The broad dips found in the near-infrared part of the SEDs can be explained by a gap in the inner part of the disks due to the presence of an embedded planet (Rice et al. 2003), or of a companion star (Marsh \& Mahoney 1992). To determine the physical size of these gaps, modelling is required. Modelling the infrared emission of each star in the binary systems will determine the infrared excess from the circumstellar disks and the contribution from the companions (Gras-Velázquez et al. 2005), as well.

We have used the luminosities and effective temperatures of the stars to place them in a H-R diagram (Fig. 15) with evolutionary tracks and isochrones from Palla \& Stahler (1999). From the H-R diagram we have estimated the masses and ages of the stars (see complete list of results in Table 5). Due to the uncertainties in both the temperatures and the luminosities, these results are not precise, although they confirm that all the objects studied are pre-main sequence (ages $\leq 10^{7} \mathrm{yr}$ ) low-mass stars $\left(0.2 \leq M_{*} / M_{\odot} \leq 2\right)$. Furthermore, there does not seem to be any clear difference between the ages of CTTS and WTTS, as already suggested by other authors, e.g. Walter (1986) or Stempels \& Piskunov (2002). It is also interesting to note that all the stars appear under the stellar birthline, considered to be the upper boundary in the H-R diagram where the $\mathrm{T}$ Tauri stars first become visible after the end of accretion of the envelope and begin their quasi-static contraction towards the ZAMS (Stahler 1983, 1988).
Table 5. Estimated ages, masses and radii of the stars in our sample and the companions.

\begin{tabular}{lcccc}
\hline \hline Star & & Age $[\mathrm{Myr}]$ & Mass $\left[M_{\odot}\right]$ & Radius $\left[R_{\odot}\right]$ \\
\hline T Cha & - & $\geq 10$ & 1.2 & 1.5 \\
Sz 68 & A & $1-3$ & 2 & 2.9 \\
& B & $\leq 30$ & $0.6-0.8$ & 0.8 \\
RU Lup & - & 1 & $0.6-0.8$ & 2.4 \\
EX Lup & - & $1-3$ & $\leq 0.6$ & 1.6 \\
Sz 124 & - & 10 & $0.6-0.8$ & 1.0 \\
& SE & - & - & - \\
ROX 2 & A & 1 & $0.6-0.8$ & $2.2(2.3)$ \\
& B & - & - & - \\
DoAr 24 & - & $1-3$ & 1.2 & 2.2 \\
DoAr 24E & A & $1-3$ & 2 & 3.5 \\
& $\mathrm{~B}$ & - & - & - \\
SR 12 & A & $\ll 1$ & $0.2-0.4$ & $2.7(2.3)$ \\
& $\mathrm{B}$ & - & - & 3.8 \\
ROX 31 & $\mathrm{A}$ & $3-5$ & $0.6-0.8$ & $1.4(2.6)$ \\
& $\mathrm{B}$ & - & - & - \\
Wa Oph/4 & $\mathrm{A}$ & $5-10$ & 1.2 & $1.6(2.4)$ \\
& $\mathrm{B}$ & - & - & - \\
Wa Oph/6 & - & 3 & $0.6-0.8$ & $1.7(3.4)$ \\
IX Oph & - & $\geq 10$ & 1.2 & 1.6 \\
V1725 Oph & - & $\geq 1$ & $\leq 0.6$ & $1.8(1.3)$ \\
& H $\alpha 2^{a}$ & 1 & $0.2-0.4$ & 1.8 \\
CoKu Ser G1 & - & 3 & $0.6-0.8$ & $1.6(2.1)$ \\
& $\mathrm{c}^{b}$ & - & - & - \\
CoKu Ser G7 & - & $1-3$ & $0.6-0.8$ & 2 \\
V536 Aq1 & $\mathrm{A}$ & $1-3$ & $0.6-0.8$ & $1.8(4.0)$ \\
& $\mathrm{B}$ & - & - & - \\
MacC H3 & - & $\geq 5$ & 1.5 & $1.8(2.6)$ \\
\hline & & & &
\end{tabular}

Notes to table: the ages and the masses have been obtained from the H-R diagram (Fig. 15). The radii using $T_{*}$ and $L_{*}$ from Table 3 . The second radius, between brackets, is the result of using alternative temperatures or luminosities suggested in Sect.3.

${ }^{a} \mathrm{~B} 62-\mathrm{H} \alpha 2$ is not really a companion, but a nearby star of V1725 Oph, associated to the same globule Barnard 62 (Reipurth \& Gee 1986; Weaver \& Jones 1992).

${ }^{b}$ Companion of CoKu Ser/G1, also known as HBC 666 (Cohen \& Kuhi 1979).

Also from the H-R diagram (see Fig. 15), we can see that the only WTTS we found with no infrared excess whatsoever, IX Oph, is one of the oldest stars in our sample.

Following Cabrit et al. (1990), we have plotted H $\alpha$ luminosity against the infrared excess for the 9 WTTS with possible circumstellar disks (see Fig. 16). Moreover in Fig. 16, we also show plots of $L_{\mathrm{H} \alpha}$ against $L_{\text {exc. and }} E W(\mathrm{H} \alpha)$ against $\mathrm{IR}_{\text {exc. }}$, for the WTTS and the CTTS in our sample, with the WTTS and CTTS from Cabrit et al. (1990). We have calculated $L_{\mathrm{H} \alpha}$ approximately from the $\mathrm{H} \alpha$ equivalent widths and the $R_{\mathrm{c}}$ magnitudes, using $L_{\mathrm{H} \alpha}=4 \pi d^{2} \cdot F_{R_{\mathrm{c}}} \cdot W_{\mathrm{H} \alpha}$ from Corcoran $\&$ Ray (1998). We have found the correlation coefficients in the four cases shown in Fig. 16 to be: 0.85 for $L_{\mathrm{H} \alpha}$ vs. $L_{\text {exc. }}$

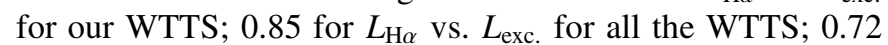
for $L_{\mathrm{H} \alpha}$ vs. $L_{\text {exc. }}$ for all WTTS and CTTS and 0.53 for $E W(\mathrm{H} \alpha)$

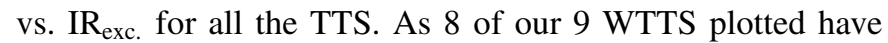
companions, the $L_{\text {exc. }}$ calculated will be an upper limit (since if 

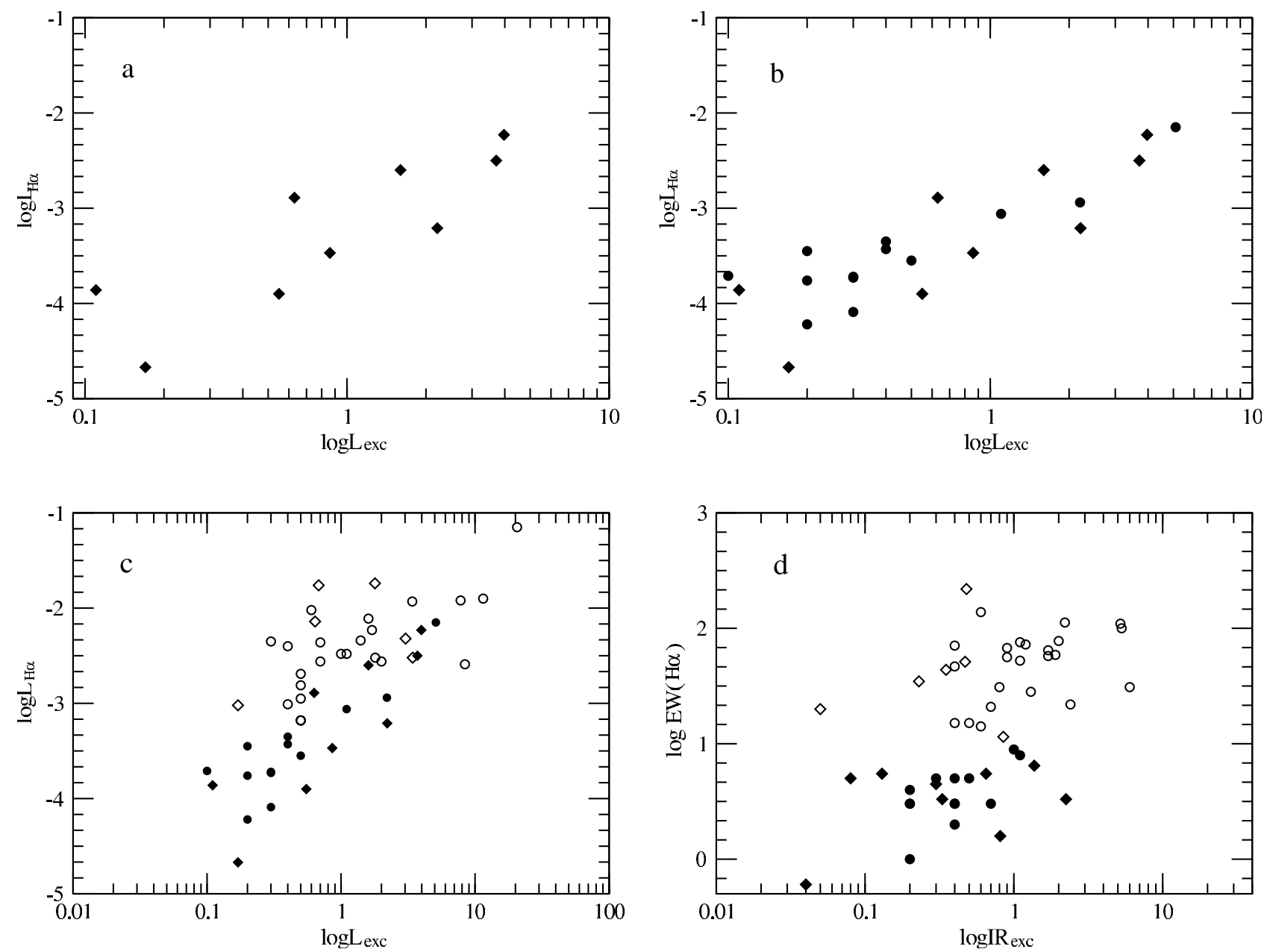

Fig. 16. In a), b) and c), $L_{\mathrm{H} \alpha}$ is in units of $L_{\odot}$. In d), $E W(\mathrm{H} \alpha)$ is in $\AA$. In the four figures: filled diamonds are the WTTS from our sample with possible circumstellar disks, filled circles are the WTTS from Cabrit et al. (1990), empty diamonds are the CTTS from our sample and empty circles are the CTTS from Cabrit et al. (1990). The Pearson product-moment correlation coefficients found between the different magnitudes are: a) 0.85 , b) 0.85 , c) 0.72 , d) 0.53 .

the contribution to the IR emission from the companions where subtracted, the $L_{\text {exc. }}$ would go down). Hence, the position of the 8 WTTS in graph c) (filled diamonds in Fig. 16) would move to the left, increasing the coherence even more. Even without shifting, there appears to be a good correlation between the $L_{\mathrm{H} \alpha}$ and the $L_{\text {exc. }}$ but not between $E W(\mathrm{H} \alpha)$ and $\mathrm{IR}_{\text {exc. }}$. Moreover the correlation coefficient found, are approximately the same as the coefficients deduced by Cabrit et al. (1990). These correlations suggest that WTTS and CTTS form a continuous spectrum and not two distinct classes divided somewhat arbitrarily by their $\mathrm{H} \alpha$ equivalent width.

\section{Conclusions}

Using photometric measurements taken by ISO, we have obtained spectral energy distributions of 6 CTTS and 12 WTTS all of which (with one exception) show an infrared excess. We have found that these excesses could indicate the presence of circumstellar disks around not only all CTTS, as expected, but also 9 of the WTTS. 8 of these WTTS plus another two WTTS also have companions or nearby stars that could contribute to the infrared excess observed, however in most cases it seems unlikely they are the dominant contributor. Moreover, half of the disks show evidence for inner dust-holes around 7.7 or $10 \mu \mathrm{m}$. Using photometry of the different stars in each system and modelling the IR excess we intend to distinguish the contribution to the IR emission from the companion and from the disks (Gras-Velázquez et al. 2005). Furthermore, the study of the characteristics of the different disk-like structures in our sample will provide information of the differences, if any, between disks around CTTS and WTTS.

The picture that emerges is one of a gradual transition in disk properties, from classical to weak-line $\mathrm{T}$ Tauri stars. The division, based on $\mathrm{H} \alpha$ equivalent widths, is somewhat arbitrary. Moreover the WTTS and the CTTS appear to be approximately coeval, suggesting that in some cases disks simply evolve faster than others. Why this should be so remains unknown.

Acknowledgements. A.G.V. warmly thanks N. Calvet, B. Merín and $\mathrm{K}$. Wood for their comments and help and also acknowledges support from Enterprise Ireland. The authors also wish to thank F. McGroarty and D. Froebrich for their help. The research has been done using the SIMBAD Database and the ALADIN image server, both operated at CDS, Strasbourg, France. 


\section{Appendix A: New photometry reduced for this paper}

In the following table we present:

a) The fluxes in Jy reduced for the stars in our sample, at each of the 9 ISOPHOT wavelengths, using PIA V10.0 and following the Ramps Pattern Mode (Gabriel et al. 1996).

b) Only the new $B, V, R$ and $I$ photometric magnitudes obtained from the observations of CoKu Ser G1, CoKu Ser G7, V536 Aql and MacC H3, taken in La Palma for this paper.

\begin{tabular}{|c|c|c|c|c|c|c|c|c|c|c|c|c|c|}
\hline & \multicolumn{9}{|c|}{$S_{v}[\mathrm{Jy}]$ at $\lambda[\mu \mathrm{m}]$} & \multicolumn{4}{|c|}{ [mag] } \\
\hline & 4.80 & 7.70 & 10.00 & 12.00 & 12.80 & 15.00 & 20.00 & 60.00 & 100.00 & $B$ & $V$ & $R_{\mathrm{c}}$ & $I_{\mathrm{c}}$ \\
\hline T Cha & 0.390 & 0.437 & 0.198 & 0.223 & 0.296 & 0.132 & 0.177 & 7.903 & 8.517 & & & & \\
\hline Sz 68 & 0.219 & 0.456 & 0.152 & 0.508 & 0.809 & 2.676 & 2.737 & 14.483 & 26.801 & & & & \\
\hline RU Lup & 0.178 & 0.140 & 0.526 & 1.053 & 0.510 & 2.228 & 2.803 & 10.017 & 7.645 & & & & \\
\hline EX Lup & 0.105 & 0.104 & 0.188 & 0.089 & - & 0.396 & 0.272 & 3.197 & 2.490 & & & & \\
\hline Sz 124 & - & - & - & - & 0.176 & - & - & 1.224 & 5.040 & & & & \\
\hline ROX 2 & - & - & - & 0.032 & 0.218 & - & - & 16.303 & 37.887 & & & & \\
\hline DoAr 24 & 0.198 & 0.063 & 0.159 & 0.232 & 0.284 & 0.157 & 0.367 & 16.237 & 72.034 & & & & \\
\hline DoAr 24E & 0.980 & 0.604 & 1.057 & 1.874 & 2.568 & 2.408 & 2.300 & 40.975 & - & & & & \\
\hline SR 12 & - & - & - & 0.030 & 0.029 & - & - & 11.710 & 49.230 & & & & \\
\hline ROX 31 & 0.168 & 0.065 & - & - & 0.268 & - & - & 1.880 & 10.230 & & & & \\
\hline $\mathrm{Wa} \mathrm{Oph/4}$ & 0.190 & - & - & 0.035 & 0.321 & - & 0.370 & 0.586 & - & & & & \\
\hline $\mathrm{Wa} \mathrm{Oph/6}$ & 0.821 & 0.757 & 0.861 & 1.390 & 1.906 & 0.613 & 1.251 & 3.896 & 5.484 & & & & \\
\hline IX Oph & 0.227 & - & - & 0.025 & 0.236 & 0.024 & - & - & - & & & & \\
\hline V1725 Oph & 0.045 & - & 0.053 & 0.085 & 0.137 & 0.062 & 0.062 & 2.424 & 7.814 & & & & \\
\hline CoKu Ser G1 & 0.111 & - & 0.480 & 0.407 & 0.586 & 0.418 & 0.512 & 4.551 & 4.883 & - & 17.4 & 16.1 & 14.2 \\
\hline CoKu Ser G7 & 0.187 & 0.214 & 0.621 & 1.391 & 0.895 & 3.300 & 2.841 & 54.967 & - & - & - & 16.4 & 14.5 \\
\hline V536 Aql & 0.218 & 0.495 & 0.628 & 1.604 & 3.332 & 2.415 & 3.650 & 8.854 & 7.353 & 15.7 & 13.9 & 12.9 & 11.4 \\
\hline $\mathrm{MacC} \mathrm{H} 3$ & - & 0.066 & 0.359 & 0.089 & 0.333 & 0.090 & - & 0.909 & - & - & - & - & 13.2 \\
\hline
\end{tabular}

\section{Appendix B: Photometry used for the SEDs from previous papers}

References for the photometry in this table can be found in Table 2 for the main stars and in the captions of Figs. 7 to 14 for the companions.

\begin{tabular}{|c|c|c|c|c|c|c|c|c|c|c|c|c|c|c|}
\hline & \multicolumn{10}{|c|}{ 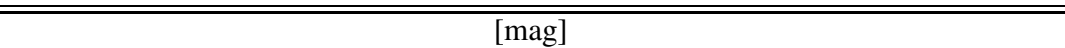 } & \multicolumn{4}{|c|}{ 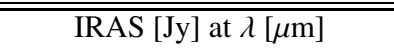 } \\
\hline & $U$ & $B$ & $V$ & $R_{\mathrm{c}}$ & $I_{\mathrm{c}}$ & $J$ & $H$ & $K$ & $L$ & $M$ & 12 & 25 & 60 & 100 \\
\hline T Cha & 13.56 & 12.65 & 11.31 & 10.51 & 9.73 & 8.96 & 7.86 & 6.95 & 5.86 & 5.39 & 0.58 & 1.74 & 6.81 & 6.46 \\
\hline Sz 68 & 12.47 & 11.58 & 10.31 & 9.56 & 8.80 & 7.57 & 6.87 & 6.48 & 6.07 & 5.40 & 2.58 & 4.08 & 7.11 & 22.61 \\
\hline RU Lup & 12.63 & 11.92 & 11.25 & 10.56 & 9.86 & 8.73 & 7.82 & 7.14 & 6.09 & - & 2.18 & 4.36 & 4.61 & 5.70 \\
\hline EX Lup & - & 13.07 & 12.34 & 11.67 & 10.88 & 9.73 & 8.96 & 8.50 & 8.05 & 7.54 & 0.80 & 1.10 & 1.25 & - \\
\hline SZ 124 & 16.08 & 14.81 & 13.33 & 12.38 & 11.46 & 10.29 & 9.63 & 9.44 & 9.31 & 9.63 & 0.10 & 0.14 & - & - \\
\hline ROX 2 & 18.90 & 17.60 & 15.40 & 13.90 & 12.30 & 9.89 & 8.75 & 8.38 & - & - & 0.20 & 0.23 & - & - \\
\hline DoAr 24 & 17.50 & 16.10 & 14.20 & 13.00 & 11.80 & 9.66 & 8.61 & 8.06 & 7.55 & - & - & - & - & - \\
\hline DoAr 24E & 17.80 & 16.80 & 14.60 & 13.06 & 11.48 & 8.97 & 7.50 & 6.57 & 5.59 & - & 5.24 & 5.91 & - & - \\
\hline SR 12 & 16.57 & 14.95 & 13.31 & 12.34 & 10.90 & 10.21 & 9.39 & 9.17 & 9.05 & - & - & - & - & - \\
\hline ROX 31 & - & 19.10 & 16.80 & 15.40 & 13.50 & 10.00 & 9.40 & 8.80 & - & - & - & - & - & - \\
\hline Wa Oph/4 & 17.25 & 15.42 & 13.52 & 12.20 & 10.99 & 9.024 & 7.930 & 7.526 & - & - & 0.62 & 0.91 & 1.08 & - \\
\hline Wa Oph/6 & 15.80 & 14.90 & 13.20 & - & - & 8.72 & 7.57 & 6.86 & - & - & 1.31 & 1.98 & 1.96 & - \\
\hline IX Oph & 13.62 & 12.80 & 11.46 & 10.66 & 9.88 & 8.57 & 8.00 & 7.72 & 7.20 & - & - & - & - & - \\
\hline V1725 Oph & - & 15.89 & 14.39 & - & - & 10.36 & 9.35 & 8.86 & - & - & - & - & - & - \\
\hline CoKu Ser G1 & - & - & - & - & - & 11.52 & 11.00 & 9.70 & - & - & 0.64 & 2.10 & 2.86 & - \\
\hline CoKu Ser G7 & - & - & 17.60 & - & - & 12.05 & 10.79 & 9.30 & - & - & 2.51 & 5.77 & 92.60 & 418.25 \\
\hline V536 Aql & - & - & - & - & - & 9.14 & 8.10 & 7.40 & 6.77 & 5.70 & 3.07 & 5.82 & 5.79 & 6.61 \\
\hline $\mathrm{MacC} \mathrm{H} 3$ & 15.44 & 15.59 & 14.50 & 14.10 & - & 11.68 & 10.99 & 10.68 & - & - & 0.27 & 0.40 & 1.66 & 2.55 \\
\hline Sz 68-B & - & - & - & 13.61 & 12.58 & 11.13 & 10.18 & 9.47 & 8.81 & 8.70 & - & - & - & - \\
\hline Sz 124SE & - & - & - & - & - & 10.82 & 9.65 & 9.15 & - & - & - & - & - & - \\
\hline ROX 2-B & - & - & - & - & - & - & - & - & - & - & - & - & - & - \\
\hline DoAr 24E-B & - & - & - & - & - & 12.10 & 9.40 & 7.81 & 5.78 & - & - & - & - & - \\
\hline SR 12-B & - & - & - & - & - & 10.97 & 10.15 & 9.93 & - & - & - & - & - & - \\
\hline ROX 31-B & - & - & - & - & - & - & - & - & - & - & - & - & - & - \\
\hline Wa Oph/4-B & - & - & - & - & 13.92 & 11.24 & 10.14 & 9.51 & - & - & - & - & - & - \\
\hline $\mathrm{B} 62-\mathrm{H} \alpha 2$ & - & - & - & - & - & 11.72 & 10.62 & 10.17 & - & - & 0.40 & 1.00 & 3.43 & 17.18 \\
\hline НBC 666 & - & - & 20.40 & 19.30 & 17.40 & 11.58 & 11.12 & 9.61 & - & - & - & - & - & - \\
\hline V536 Aql-B & - & - & - & - & - & 10.89 & 9.38 & 8.71 & 7.94 & 6.30 & - & - & - & - \\
\hline
\end{tabular}

Note to Tables A and B: the photometry presented here is uncorrected for extinction. 


\section{References}

Ageorges, N., Méarned, F., Monin, J.-L., \& Eckart, A. 1994, A\&A, 283, L5

Ageorges, N., Eckart, A., Monin, J.-L., \& Ménard, F. 1997, A\&A, 326,632

Alcalá, J. M., Covino, E., Franchini, et al. 1993, A\&A, 272, 225

Appenzeller, I., Krautter, J., \& Jankovics, I. 1983, A\&AS, 53, 291

Barsony, M., Koresko, C., \& Matthews, K. 2003, ApJ, 591, 1064

Batalha, C. C., Ouast, G. R., Torres, C. A. O., et al. 1998, A\&AS, 128, 561

Bertin, E., \& Arnouts, S. 1996, A\&AS, 117, 393

Bessell, M. S. 1979, PASP, 91, 589

Bertout, C., Robichon, N., \& Arenou, F. 1999, A\&A, 352, 574

Bouvier, J. \& Appenzeller, I. 1992, A\&AS, 92, 481

Brown, D. W., Chandler, C. J., Carlstrom, J. E., et al. 2000, MNRAS, 319, 154

Cabrit, S., Edwards, S., Strom, S. E., \& Strom, K. M. 1990, ApJ, 354, 687

Calvet, N., D'Alessio, P., Hartmann, L., et al. 2002, ApJ, 568, 1008

Chandler, C. J. 1998, ASP Conf. Ser. 148: Origins, 237

Chavarría-K. C. 1981, A\&A, 101, 105

Chavarría-K. C., Terranegra, L., Moreno-Corral, M. A., \& de Lara, E. 2000, A\&AS, 145, 187

Chelli, A., Cruz-González, I., Zinnecker, H., Carrasco, L., \& Perrier, C. $1988, A \& A, 207,46$

Chini, R., \& Wargau, W. F. 1998, A\&A, 329, 161

Cohen, M., \& Kuhi, L. V. 1979, ApJS, 41, 743

Corcoran, M., \& Ray, T. P. 1998, A\&A, 331, 147

Covino, E., Terranegra, L., Franchini, M., Chavarría-K., C., \& Stalio, R. 1992, A\&AS, 94, 273

de Zeeuw, P. T., Hoogerwerf, R., de Bruijne, J. H. J., Brown, A. G. A., \& Blaauw, A. 1999, AJ, 117, 354

Duvert, G., Guilloteau, S., Ménard, F., Simon, M., \& Dutrey, A. 2000, A\&A, 355, 165

Favata, F., Micela, G., Sciortino, S., \& D’Antona, F. 1998, A\&A, 335, 218

Fernández, M., Ortiz, E., Eiroa, C., \& Miranda, L. F. 1995, A\&AS, 114,439

Finkenzeller, U., \& Basri, G. 1987, ApJ, 318, 823

Gabriel, C., Haas, M., Heinrichsen, I., \& Tai, W. M. 1996, ISOPHOT Interactive Analysis User Manual, available from ESA/ISO Science Operations Centre, VILSPA, or MPI Astronomie Heidelberg

Gahm, G. F., Gullbring, E., Fischerstrom, C., Lindroos, K. P., \& Loden, K. 1993, A\&AS, 100, 371

Geoffray, H., \& Monin, J.-L. 2001, A\&A, 369, 239

Ghez, A. M., McCarthy, D. W., Patience, J. L., \& Beck, T. L. 1997, ApJ, 481, 378

Glass, I. S., \& Penston, M. V. 1974, MNRAS, 167, 237

Gras-Velázquez, À., Walker, C. H., Ray, T. P., \& Wood, K. 2005, in preparation
Günther, R., \& Kley, W. 2002, A\&A, 387, 550

Herbig, G. H., Gilmore, A. C., \& Suntzeff, N. 1992, Informational Bulletin on Variable Stars, 3808, 1

Herbig, G. H., Aspin, C., Gilmore, A. C., Imhoff, C. L., \& Jones, A. F. 2001, PASP, 113, 1547

Hillenbrand, L. A., Strom, S. E., Vrba, F. J., \& Keene, J. 1992, ApJ, 397,613

Hirth, G. A., Mundt, R., \& Solf, J. 1997, A\&AS, 126, 437

Hughes, J., Hartigan, P., Krautter, J., \& Kelemen, J. 1994, AJ, 108, 1071

Jensen, E. L. N., Mathieu, R. D., \& Fuller, G. A. 1996, ApJ, 458, 312

Jensen, E. L. N., Mathieu, R. D., Donar, A. X., \& Dullighan, A. 2004, ApJ, 600, 789

Kenyon, S. J., \& Hartmann, L. 1987, ApJ, 323, 714

Kenyon, S. J., \& Hartmann, L. W. 1990, ApJ, 349, 197

Kraemer, K. E., Shipman, R. F., Price, S. D., et al. 2003, AJ, 126, 1423

Lang, K.R. 1992, Astrophysical Formulae: A Compendium for the Physicist and Astrophysicist (Springer-Verlag Berlin and Heidelberg GmbH \& Co. K)

Littlefair, S. P., Naylor, T., Harries, T. J., Retter, A., \& O'Toole, S. 2004, MNRAS, 347, 937

Marsh, K. A., \& Mahoney, M. J. 1992, ApJ, 395, L115

Martín, E. L. 1998, AJ, 115, 351

Meyer, M. R., Beckwith, S. V. W., \& Natta, A. 1997, Herbig-Haro Flows and the Birth of Stars, IAU Symp., 182, 224

Montmerle, T., Koch-Miramond, L., Falgarone, E., \& Grindlay, J. E. 1983, ApJ, 269, 182

Mundt, R., \& Bastian, U. 1980, A\&AS, 39, 245

Mundt, R., \& Eislöffel, J. 1998, AJ, 116, 860

Palla, F., \& Stahler, S. W. 1999, ApJ, 525, 772

Reid, N. 2002, http://www. stsci . edu/ inr/cmd.html

Reipurth, B., \& Gee, G. 1986, A\&A, 166, 148

Reipurth, B., Nyman, L.-A., \& Chini, R. 1996, A\&A, 314, 258

Rice, W. K. M., Wood, K., Armitage, P. J., Whitney, B. A., \& Bjorkman, J. E. 2003, MNRAS, 342, 79

Rydgren, A. E. 1980, AJ, 85, 438

Reipurth, B., \& Zinnecker, H. 1993, A\&A, 278, 81

Rydgren, A. E., Strom, S. E., \& Strom, K. M. 1976, ApJS, 30, 307

Schwartz, R. D., \& Noah, P. 1978, AJ, 83, 785

Siebenmorgen, R., Prusti, T., Natta, A., \& Müller, T. G. 2000, A\&A, 361,258

Simon, M., Howell, R. R., Longmore, A. J., et al. 1987, ApJ, 320, 344

Stahler, S. W. 1983, ApJ, 274, 822

Stahler, S. W. 1988, ApJ, 332, 804

Stempels, H. C., \& Piskunov, N. 2002, A\&A, 391, 595

Teixeira, T. C., \& Emerson, J. P. 1999, A\&A, 351, 292

VandenBerg, D. A., \& Bridges, T. J. 1984, ApJ, 278, 679

Walter, F. M. 1986, ApJ, 306, 573

Weaver, W. B., \& Jones, G. 1992, ApJS, 78, 239

Wood, K., Lada, C. J., Bjorkman, J. E., et al. 2002, ApJ, 567, 1183 